\title{
BLACK HOLE AND NEUTRON STAR TRANSIENTS IN QUIESCENCE
}

\author{
Kristen Menou, ${ }^{1,2}$ Ann A. Esin, ${ }^{3,4}$ Ramesh Narayan, ${ }^{1}$ Michael R. Garcia, ${ }^{1}$ \\ JeAN-Pierre Lasota, ${ }^{2,5}$ AND JefFrey E. McClintock ${ }^{1}$ \\ Received 1998 October 16; accepted 1999 February 24
}

\begin{abstract}
We consider the X-ray luminosity difference between neutron star and black hole soft X-ray transients (NS and BH SXTs) in quiescence. The current observational data suggest that BH SXTs are significantly fainter than NS SXTs. The luminosities of quiescent BH SXTs are consistent with the predictions of binary-evolution models for the mass transfer rate if (1) accretion occurs via an advection-dominated accretion flow (ADAF) in these systems and (2) the accreting compact objects have event horizons. The luminosities of quiescent NS SXTs are not consistent with the predictions of ADAF models when combined with binary-evolution models, unless most of the mass accreted in the ADAF is prevented from reaching the neutron star surface. We consider the possibility that mass accretion is reduced in quiescent NS SXTs because of an efficient propeller and develop a model of the propeller effect that accounts for the observed luminosities. We argue that modest winds from ADAFs are consistent with the observations, while strong winds are probably not.

Subject headings: accretion, accretion disks — binaries: close — black hole physics stars: magnetic fields - stars: neutron - X-rays: stars
\end{abstract}

\section{INTRODUCTION}

Soft X-ray transients (SXTs) are compact binary systems in which a low-mass secondary (either a main-sequence star or a subgiant) transfers mass via Roche-lobe overflow onto a black hole (BH) or neutron star (NS) primary (see reviews by Tanaka \& Lewin 1995; van Paradijs \& McClintock 1995; White, Nagase, \& Parmar 1995). SXTs have highly variable luminosities. They spend most of their lifetimes in a low-luminosity quiescent state, but occasionally undergo dramatic outbursts during which both the optical and X-ray emission increase by several orders of magnitude (see, e.g., Chen, Shrader, \& Livio 1997a; Kuulkers 1998). NS SXT outbursts typically occur every 1-10 yr and last for several weeks, while BH SXT outbursts are typically separated by $10-50 \mathrm{yr}$ (or perhaps longer) and last for several months (see Chen et al. 1997a).

A variety of observations (see, e.g., Tanaka \& Shibazaki 1996) indicate that, near the peak of an outburst, an SXT accretes matter via a standard thin disk (Shakura \& Sunyaev 1973), so that there is little doubt that the accretion is radiatively efficient during this phase. The situation is more complex in quiescence. The spectra of quiescent $\mathrm{BH}$ SXTs do not resemble that of a thin disk, and the accretion rates inferred from the observed X-ray luminosities disagree by orders of magnitude with the predictions of the standard disk-instability model for quiescent disks (see, e.g., Lasota 1996).

\footnotetext{
${ }^{1}$ Harvard-Smithsonian Center for Astrophysics, 60 Garden Street, Cambridge, MA 02138; kmenou@cfa.harvard.edu, rnarayan@ cfa.harvard.edu,mgarcia@cfa.harvard.edu,jmcclintock@cfa.harvard.edu.

${ }^{2}$ Unité Propre de Recherche 176 du Centre National de la Recherche Scientifique, Département d'Astrophysique Relativiste et de Cosmologie, Observatoire de Paris, Section de Meudon, 92195 Meudon Cédex, France.

3 Theoretical Astrophysics, Caltech 130-33, Pasadena, CA 91125; aidle@tapir.caltech.edu.

${ }^{4} A X A F$ Fellow.

${ }^{5}$ Present address: Institut d'Astrophysique de Paris, 98bis Boulevard Arago, 75014 Paris, France; lasota@iap.fr.
}

Narayan, McClintock, \& Yi (1996) and Narayan, Barret, $\&$ McClintock (1997a) showed that the observations of quiescent BH SXTs can be explained by a two-component accretion flow model consisting of an inner hot advectiondominated accretion flow (ADAF; Narayan \& Yi 1994, 1995a; Abramowicz et al. 1995; Ichimaru 1977; see Narayan, Mahadevan, \& Quataert 1999 and Kato, Fukue, \& Mineshige 1998 for reviews of ADAFs) surrounded by an outer thin disk. In the recent version of this model described in Narayan et al. (1997a), only the inner ADAF contributes to the observed optical, UV, and X-ray emission of the system. The outer thin disk acts mainly as a reservoir that accumulates mass until the next outburst is triggered. In quiescence, the emission of the disk is primarily in the infrared; this radiation is hardly seen as it is swamped by the emission of the secondary (see Narayan et al. 1996, 1997a; Lasota, Narayan, \& Yi 1996 for details).

A key feature of the Narayan et al. $(1996,1997 a)$ model of quiescent BH SXTs is the low radiative efficiency of the ADAF. In these flows, the bulk of the viscously dissipated energy is stored in the gas and advected with the flow into the black hole (Narayan \& Yi 1995b; Abramowicz et al. 1995; Narayan et al. 1996, 1997a). This explains the unusually low luminosities of BH SXTs. By contrast, in NS SXTs all the advected energy is expected to be radiated from the neutron star surface, resulting in a much higher radiative efficiency of the accretion flow even in the presence of an ADAF (Narayan \& Yi 1995b). Motivated by this fundamental distinction between black hole and neutron star systems, Narayan, Garcia, \& McClintock (1997b) and Garcia et al. (1998) compared the outburst amplitudes of BH SXTs and NS SXTs as a function of their maximum luminosities and showed that the observations reveal systematically lower relative luminosities in BH SXTs. They argued that this constitutes a confirmation of the presence of an event horizon in BH SXTs. The argument was challenged by Chen et al. (1998).

In this paper, we attempt to develop a physical understanding of the difference in quiescent luminosities between 
NS and BH SXTs. We first show, in $\S 2$, that there is indeed a significant difference in the observed quiescent luminosities of the two classes of objects, in contrast to the claim of Chen et al. (1998). We then use binary-evolution models in $\S 3$ to estimate mass transfer rates in SXTs. We combine these estimates with the ADAF + thin disk accretion scenario, taking into account the presence of an event horizon in BH systems and a reradiating surface in NS systems, to determine the expected X-ray luminosities in quiescence $(\S 4)$. The model predictions agree well with the observations of quiescent BH SXTs. However, the model substantially overestimates the luminosities of quiescent NS SXTs. In $\S 5$ we show that the NS SXT data can be reconciled with the predictions of the model if we take into account the "propeller effect" (Illarionov \& Sunyaev 1975), whereby the magnetosphere of a rapidly rotating neutron star prevents much of the accreting material from reaching the surface of the neutron star. In $\S 6$ we show that an ADAF model with a moderate wind and a somewhat less efficient propeller (in NS SXTs) is also consistent with the observed quiescent luminosities of BH and NS SXTs. Finally, in $\S 7$ we discuss possible limitations and extensions of this work and in $\S 8$ we summarize the main results.

\section{OBSERVATIONS}

In Table 1, we list key parameters of several NS and BH SXTs: the orbital period $P_{\text {orb }}$, the distance $D$, the quiescent X-ray luminosity $L_{\min }$ in the $0.5-10 \mathrm{keV} \mathrm{X-ray} \mathrm{band,} \mathrm{and}$ the mass $m_{1}$ (in solar units) of the compact primary. Since our main interest is in the quiescent emission, we list only systems for which there exist reliable measurements of $L_{\min }$. Finally, the ratio $L_{\min } / L_{\max }$ (which is independent of the distance $D$ ) is used for comparison with previous investigations of the luminosity difference between $\mathrm{BH}$ and NS SXTs. The values of $L_{\max }$, the outburst peak luminosity, are taken from Garcia et al. (1998). Here, we choose to ignore $L_{\max }$, which depends mainly on the physics of the thin disk in outburst, and concentrate just on $L_{\min }$, which characterizes the quiescent state of an SXT. The values of $L_{\min }$ are taken from Narayan et al. (1997b) and Garcia et al. (1998), except for the BH SXT H1705-250 (see below).

In selecting the systems listed in Table 1 , we were careful to avoid any possible confusion between BH and NS SXTs, since this would make a comparison between the two sets of objects less reliable. Thus, we limit our sample to the eight $\mathrm{BH}$ candidates that have firm dynamical lower limits on the

TABLE 1

NeUtron Star and Black Hole SXTs

\begin{tabular}{|c|c|c|c|c|}
\hline $\begin{array}{l}\text { System } \\
\text { (1) }\end{array}$ & $\begin{array}{c}P_{\text {orb }} \\
(\mathrm{hr}) \\
(2)\end{array}$ & $\begin{array}{c}D \\
(\mathrm{kpc}) \\
(3)\end{array}$ & $\begin{array}{c}\log L_{\min } \\
\left(\mathrm{ergs} \mathrm{s}^{-1}\right) \\
(4)\end{array}$ & $\begin{array}{c}m_{1} \\
\left(M_{\odot}\right) \\
(5)\end{array}$ \\
\hline \multicolumn{5}{|c|}{ NS Primary } \\
\hline EXO $0748-676 \ldots \ldots \ldots \ldots \ldots \ldots$ & $3.82^{\mathrm{a}}$ & $10^{\mathrm{b}}$ & 34.1 & $1.4^{\mathrm{c}}$ \\
\hline $4 \mathrm{U} 2129+47 \ldots \ldots \ldots \ldots \ldots \ldots \ldots$ & $5.2^{\mathrm{d}}$ & $6.3^{\mathrm{b}}$ & 32.8 & $1.4^{\mathrm{c}}$ \\
\hline $1456-32($ Cen X-4) ................ & $15.1^{\mathrm{a}}$ & $1.2^{\mathrm{b}}$ & 32.4 & $1.4^{\mathrm{c}}$ \\
\hline $1908+005($ Aq1 X-1) ............... & $19^{\mathrm{a}}$ & $2.5^{\mathrm{b}}$ & 32.6 & $1.4^{\mathrm{c}}$ \\
\hline $\mathrm{H} 1608-52 \ldots \ldots \ldots \ldots \ldots \ldots \ldots \ldots \ldots \ldots \ldots \ldots \ldots$ & $98.4^{e}$ or $5^{f}$ & $3.6^{\mathrm{b}}$ & 33.3 & $1.4^{\mathrm{c}}$ \\
\hline \multicolumn{5}{|c|}{ BH Primary } \\
\hline GRO J0422+ 32 (XN Per 92) ...... & 5.1 & $2.6^{\mathrm{g}}$ & $<31.6$ & $12^{\mathrm{h}}$ \\
\hline A0620-00 (XN Mon 75) .......... & 7.8 & $1^{\mathrm{i}}$ & 31.0 & $6.1^{\mathrm{i}}$ \\
\hline GS2000+25 (XN Vul 88) ......... & 8.3 & $2.7^{\mathrm{i}}$ & $<32.3$ & $8.5^{\mathrm{j}}$ \\
\hline GS $1124-683(\mathrm{XN}$ Mus 91$) \ldots \ldots$ & 10.4 & $5^{\mathrm{k}}$ & $<32.4$ & $6^{\mathrm{k}}$ \\
\hline H1705-250 (XN Oph 77) ......... & 12.5 & $8.6^{\mathrm{i}}$ & $<33.0$ & $4.9^{\mathrm{j}}$ \\
\hline $4 \mathrm{U} 1543-47 \ldots \ldots \ldots \ldots \ldots \ldots \ldots$ & 27.0 & $8^{\mathrm{b}}$ & $<33.3^{1}$ & $7^{\mathrm{m}}$ \\
\hline J1655-40 (XN Sco 94) ............ & 62.9 & $3.2^{\mathrm{n}}$ & 32.4 & $7^{\mathrm{n}}$ \\
\hline GS $2023+338$ (V404 Cyg) ......... & 155.3 & $3.5^{\mathrm{i}}$ & 33.2 & $12^{\mathrm{i}}$ \\
\hline
\end{tabular}

Notes.-Col. (2): Orbital periods from McClintock 1998, except where indicated. Col. (3): Distances to the systems. Col. (4): Luminosities in quiescence in the $0.5-10 \mathrm{keV}$ band (corrected for the revised distances) from Narayan et al. 1997b and Garcia et al. 1998, except where indicated. Quiescent luminosities for 4U 1543-47 and GRO J165540 are based on $20.4 \mathrm{ks}$ and $100 \mathrm{ks} A S C A$ observations (respectively), and the quiescent luminosity for GRO J0422+32 is based on a 19 ks ROSAT observation. Col. (5): Primary masses.

a van Paradijs 1995.

${ }^{\mathrm{b}}$ Garcia et al. 1998.

c For simplicity, all NS masses are assumed to be $1.4 M_{\odot}$.

d Simbad CDS Catalog.

e Ritter \& Kolb 1998.

${ }^{\mathrm{f}}$ Chen et al. 1998.

${ }^{\mathrm{g}}$ Esin et al. 1998.

${ }^{\mathrm{h}}$ Beekman et al. 1997.

i Narayan et al. 1997b.

${ }^{j}$ Chen et al. 1997a.

${ }^{k}$ Esin et al. 1997.

${ }^{1}$ Orosz et al. 1998.

$\mathrm{m}$ This is an arbitrary choice in the range $2.9-7.5$ given by Orosz et al. 1998; see also Bailyn et al. 1998.

${ }^{\mathrm{n}}$ Hameury et al. 1997. 

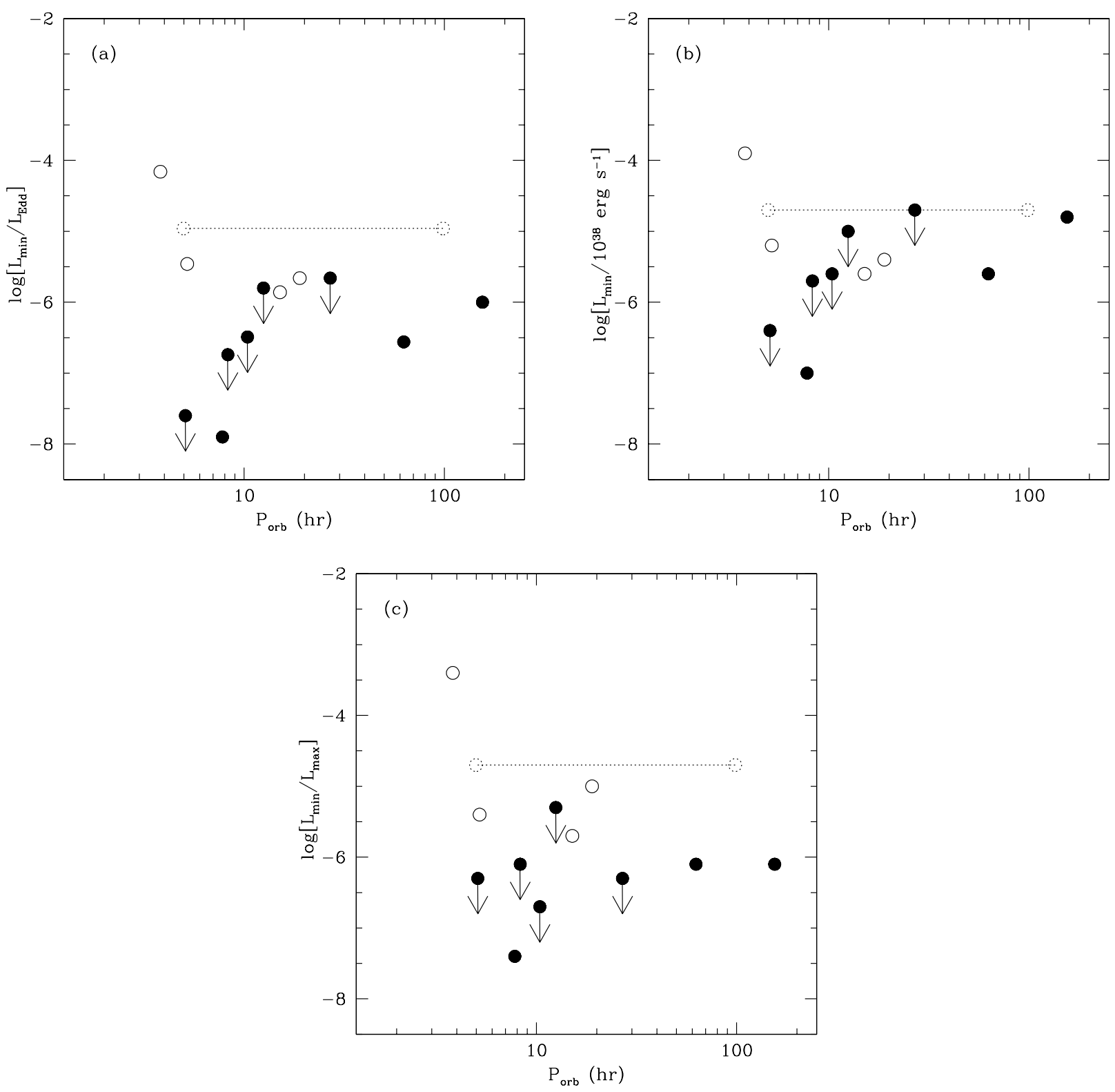

FIG. 1.- (a) Quiescent luminosities $L_{\min }$ (Eddington units) in the $0.5-10 \mathrm{keV}$ band of the NS SXTs (open circles) and BH SXTs (filled circles) listed in Table 1. The luminosities are plotted as a function of the orbital period $P_{\text {orb }}$ of each system. (b) Same as $(a)$ except that $L_{\min }$ are shown in units of $10^{38}$ ergs $\mathrm{s}^{-1}$. (c) Same as $(a)$, except that the ratio $L_{\min } / L_{\max }$ is shown.

mass of the primary (see, e.g., McClintock 1998). (Note that this is not true for several so-called BH "candidates" of Chen et al. 1998, which seriously weakens their arguments.) Similarly, there is firm evidence that the five systems listed in Table 1 as NS SXTs contain NS primaries, based on the detection of type I X-ray bursts (see, e.g., Narayan et al. 1997b; Chen et al. 1997a).

Here we report a new and improved luminosity limit for H1705-250 (= Nova Oph 77) based on the $A S C A$ observation of 1996 September 18, which we extracted from the HEASARC archive. The exposure time was $31 \mathrm{ks}$, but unfortunately the target was near the chip boundaries, which limits the effective area. The $4 \sigma$ upper limit is $0.9 \times 10^{-3}$ counts $\mathrm{s}^{-1}$, which corresponds to an X-ray flux $(1-40 \mathrm{keV})<1 \times 10^{-13} \mathrm{ergs} \mathrm{s}^{-1} \mathrm{~cm}^{-2}$. Assuming a distance of $8.6 \mathrm{kpc}, L_{\mathrm{X}}<0.9 \times 10^{33} \mathrm{ergs} \mathrm{s}^{-1}$.

Luminosities can be expressed in units of the Eddington luminosity,

$$
L_{\text {Edd }}=1.25 \times 10^{38} m_{1} \operatorname{ergs~s}^{-1},
$$

where $m_{1}$, the mass of the compact object in solar units, is listed in Table 1. For a standard radiative efficiency of $10 \%$, the Eddington luminosity corresponds to a mass accretion rate of

$$
\dot{M}_{\text {Edd }}=1.39 \times 10^{18} m_{1} \mathrm{~g} \mathrm{~s}^{-1} .
$$

In this section, we use both absolute luminosities and Eddington-scaled luminosities ${ }^{6}$ since it is not clear which is the more appropriate quantity for comparisons. Our calculations in $\S 3$ suggest that the mass transfer rates in NS and

\footnotetext{
${ }^{6}$ Note that Eddington-scaled luminosities are somewhat uncertain if the primary mass $m_{1}$ in a system is not well known (eq. [1]). This is, for instance, the case of the BH SXT J1655-40. Orosz \& Bailyn (1997) estimate that $m_{1}=7$ in this system, while Phillip, Shahbaz, \& Podsiadlowski (1999) claim that $m_{1}$ could be as low as 4.1.
} 

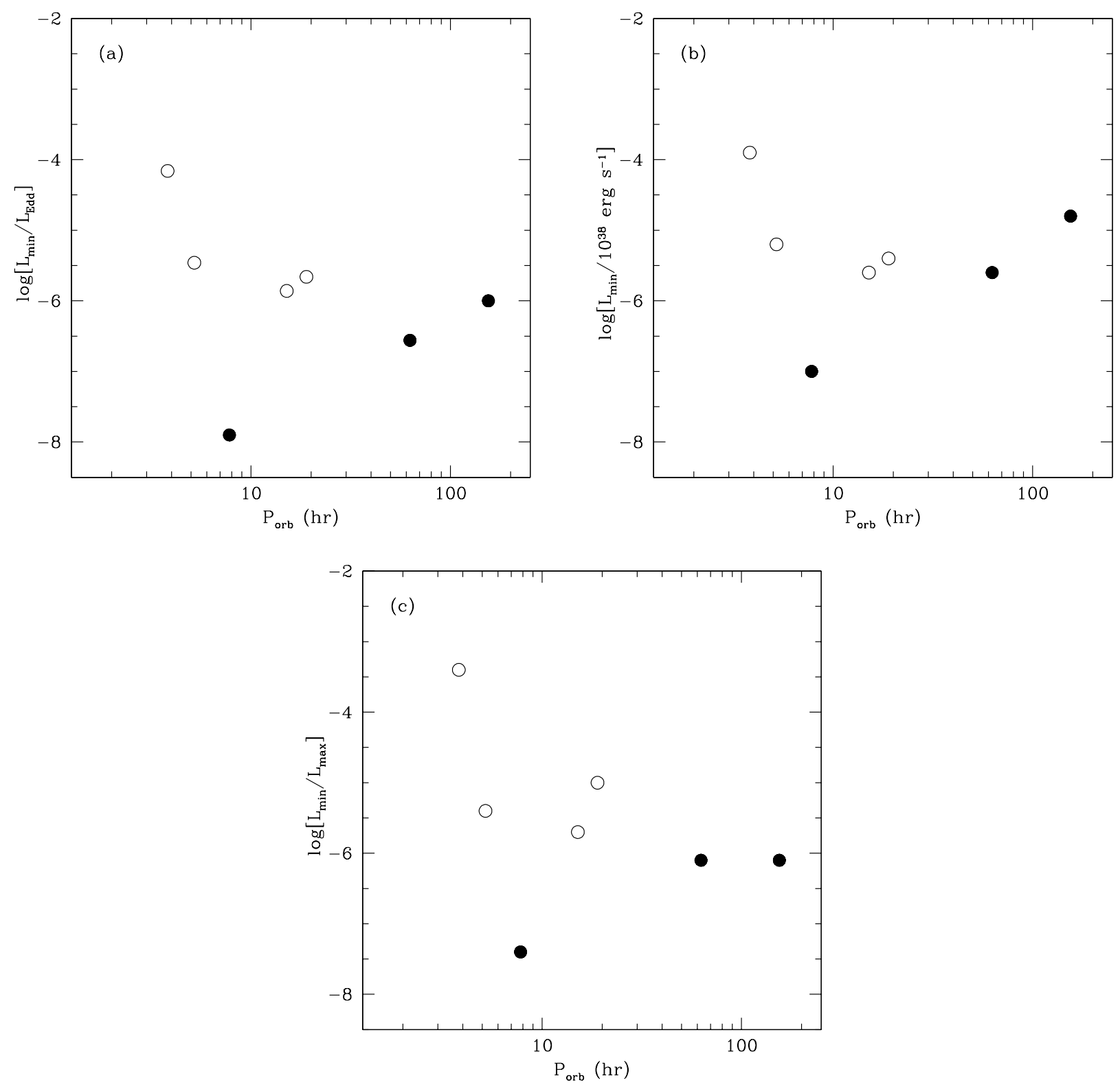

FIG. 2. - (a) Same as Fig. $1 a$, except that only SXTs with well-determined $L_{\min }$ and $P_{\text {orb }}$ are shown. (b) Same as $(a)$ except that $L_{\min }$ are shown in units of $10^{38} \mathrm{ergs} \mathrm{s}^{-1}$. (c) Same as $(a)$, except that the ratio $L_{\min } / L_{\max }$ is shown.

BH SXTs are similar when expressed in units of $\dot{M}_{\text {Edd }}$, especially at short orbital periods. Thus, Eddington-scaled luminosities might be more appropriate to compare the two classes of objects at short periods (where most of the data lie). However, this argument is not very strong. Therefore, we also show absolute luminosities, expressed in units of $10^{38} \mathrm{ergs} \mathrm{s}^{-1}$ (which is roughly the luminosity expected from an object accreting at $10^{18} \mathrm{~g} \mathrm{~s}^{-1}$ with a radiative efficiency of $10 \%$ ).

In Figure 1, we show the quiescent luminosities of the $\mathrm{BH}$ and NS systems listed in Table 1 as a function of their orbital periods $P_{\text {orb }}$. The open circles correspond to NS SXTs and the filled dots correspond to BH systems. Luminosity upper limits (indicated by downward arrows) are shown for five SXTs, all of which are BH systems. The orbital period of the NS SXT H1608-52 is uncertain (see Table 1), and its location in Figure 1 is indicated by dashed circles. Among the undetected SXTs, we choose to include in our sample only those systems that have been observed for more than $10 \mathrm{ks}$ (see Table 1 herein, and also Table 1 in Narayan et al. 1997b), which therefore have flux limits $\lesssim 3$ $\times 10^{-13} \mathrm{ergs} \mathrm{s}^{-1}$ with current X-ray satellites $(0.5-10 \mathrm{keV}$; ROSAT/ASCA).

The argument for using $P_{\text {orb }}$ along the horizontal axis of Figure 1 is as follows. For any binary system with a lowmass secondary, and transferring mass via Roche-lobe overflow, the density of the secondary essentially determines $P_{\text {orb }}$. At a given $P_{\text {orb }}$, a BH SXT and an NS SXT will have similar secondaries, so that the mass transfer characteristics are likely to be similar. Thus a reliable comparison of the radiative efficiencies of their accretion flows would be possible. In contrast, if we were to compare the quiescent luminosities of a BH SXT and an NS SXT with quite different $P_{\text {orb }}$, a difference in the mass transfer rates (see $\S 3$ ) could mask actual differences in the radiative efficiencies of the accretion flows. This point, which is the motivation for the calculations in $\S 3$, was first emphasized by Lasota \& Hameury (1998). 
Figure 1 strongly suggests that BH SXTs are fainter than NS SXTs. This is especially true when the data are plotted in Eddington units (Fig. 1a), but it is reasonably convincing even in absolute units (Fig. 1b) or in terms of the ratio $L_{\min } / L_{\max }$ (Fig. 1c). Note that all five of the NS SXTs have been detected in quiescence and have well-measured values of $L_{\min }$, while the majority of BH SXTs have only upper limits on $L_{\min }$. In our view, this is significant and underlines the effect we are claiming.

To make a clearer comparison between BH and NS SXTs with the existing data, we isolate in Figure 2 those systems for which $L_{\min }$ and $P_{\text {orb }}$ are well determined (see also the very similar Fig. 3 of Lasota \& Hameury 1998). Although there are only a few systems in this figure, we feel that we can conclude that (at least in this small sample) BH SXTs in quiescence are less luminous than NS SXTs. The difference is as much as $\mathbf{2}$ orders of magnitude (in Eddington units) for short-period systems.

It is important to stress here the excellent upper limit on the quiescent luminosity of the BH SXT J0422+32. This upper limit is nearly equal to the very low measured quiescent luminosity of A0620-00. Thus, there are now two BH SXTs that are 2 orders of magnitude less luminous, in Eddington units, than quiescent NS SXTs with comparable $P_{\text {orb }}$. It is important to carry out more sensitive observations of those BH SXTs that have only upper limits currently.

Narayan et al. (1997b) explained the difference in quiescent luminosities of NS SXTs and BH SXTs as due to the presence of a hard surface in NS SXTs versus an event horizon in BH SXTs. The presence of a surface causes the radiative efficiency in NS SXTs to be high, while in $\mathrm{BH}$ SXTs most of the dissipated energy is advected into the event horizon of the $\mathrm{BH}$, without producing observable emission. Narayan et al. argued that this constitutes observational evidence for event horizons in black holes. In the remainder of the paper, we expand on this idea and construct a physical model of accretion in NS and BH systems to explain quantitatively the observations summarized in Figure 2. In $\S 3$ we estimate the mass transfer rate in SXTs, and in $\S \S 4$ and 5 we describe an accretion model that is able to reproduce the observed emission in the $0.5-10 \mathrm{keV}$ band.

\section{MASS TRANSFER RATES PREDICTED BY BINARY-EVOLUTION MODELS}

The theory of binary evolution relies on the Roche-lobe model for the description of mass transfer (Frank, King, \& Raine 1992). Depending on which mechanism drives the mass transfer, whether it is loss of orbital angular momentum through gravitational radiation and magnetic braking, or expansion of the donor as it evolves away from the main sequence, the binaries are classified as $j$-driven or $n$-driven systems. There is a "bifurcation" orbital period $P_{\text {bif }}$ separating the two classes such that for $P_{\text {orb }}>P_{\text {bif }}$ we find $n$-driven systems whose orbital periods increase with time, and for $P_{\text {orb }}<P_{\text {bif }}$ we find $j$-driven systems whose $P_{\text {orb }}$ decrease with time. Although there is no unique value of $P_{\text {bif }}$, because it depends on the donor mass and the strength of angular momentum losses, estimates range from 0.5-2 days (see, e.g., Pylyser \& Savonije 1988; King, Kolb, \& Burderi 1996).

The exact form of the magnetic braking law is not well established (see, e.g., Kalogera, Kolb, \& King 1998). Moreover, Menou, Narayan, \& Lasota (1999) showed that there is strong circumstantial evidence that magnetic braking (MB) is weak in BH SXTs. In the following, for simplicity, we neglect the influence of MB in BH and NS SXTs. We will see in $\S 4.2$ that any contribution to the mass transfer by MB would only strengthen the argument that NS SXTs shed mass rather than accrete it.

Following King et al. (1996), the mass transfer rates in $j$-driven systems with gravitational radiation (GR) and $n$ driven system with secondary expansion (EXP) is given by

$$
\begin{aligned}
\dot{M}_{T} \approx & 1.27 \times 10^{14} m_{1} m_{2}^{2}\left(m_{1}+m_{2}\right)^{-1 / 3} \\
& \left(P_{\text {orb }} / 1 \text { day }\right)^{-8 / 3} \mathrm{~g} \mathrm{~s}^{-1}, \\
& P_{\text {orb }}<P_{\text {bif }}(\mathrm{GR}) ; \\
\approx & 2.54 \times 10^{16} m_{2}^{1.47}\left(P_{\text {orb }} / 1 \text { day }\right)^{0.93} \mathrm{~g} \mathrm{~s}^{-1}, \\
& P_{\text {orb }}>P_{\text {bif }}(\mathrm{EXP}) ;
\end{aligned}
$$

where $m_{2}$ is the mass of the secondary in solar units and $P_{\text {orb }}$ is scaled in units of 1 day.

The secondary in $j$-driven binaries is usually a mainsequence star (see King et al. 1996 for possible complications) filling its Roche lobe. These stars obey the simple relation $m_{2}=0.11\left(P_{\text {orb }} / 1 \mathrm{hr}\right.$ ) (see, e.g., Frank et al. 1992). The prediction for $\dot{M}_{T}$ in case of GR-driven mass transfer then becomes

$$
\begin{aligned}
\dot{M}_{T} \approx & 8.85 \times 10^{14} m_{1}\left(m_{1}+m_{2}\right)^{-1 / 3} \\
& \left(P_{\text {orb }} / 1 \text { day }\right)^{-2 / 3} \mathrm{~g} \mathrm{~s}^{-1}, \\
& P_{\text {orb }}<P_{\text {bif }}(\mathrm{GR}),
\end{aligned}
$$

which scales with the mass of the primary as $m_{1}^{2 / 3}$ for small $m_{2}$. Note this is not very different from $\dot{M}_{T} \propto m_{1}$, thus motivating the use of the Eddington scaling of luminosity in Figures 1 and 2.

Equation (4) for the case of secondary expansion is the linear limit of third-order polynomial fits (King 1988) to detailed evolutionary tracks of single stars computed by Webbink, Rappaport, \& Savonije (1983). Along an evolutionary track, the mass $m_{2}$ of the expanding secondary decreases as it transfers mass onto the primary. Since a variety of initial masses and orbital periods (at the onset of mass transfer) are possible, $m_{2}$ is essentially a free parameter in equation (4). However, a careful study of evolutionary tracks ${ }^{7}$ shows that the band of mass transfer rates predicted by equation (4) for values of $m_{2}$ in the range 0.5-1 reproduces the mass transfer rates in $n$-driven accreting binaries for the majority of their lifetime. This band therefore provides, in a "population synthesis" sense, the likely mass transfer rates one can expect in SXTs with orbital periods larger than the bifurcation period.

We estimate the mass transfer rate expected at a given orbital period by simply adding the contributions from GR (eq. [5]) and secondary expansion (a band with $m_{2}=$ $[0.5,1]$ in eq. [4]). Figure 3 shows that this results in a relatively short, although plausible, bifurcation period of $\sim 10 \mathrm{hr}$. The estimates of $M_{T}$ are shown separately for BH and NS SXTs, in units of $10^{18} \mathrm{~g} \mathrm{~s}^{-1}$. The mass transfer rates are typically similar, in absolute units, in BH and NS SXTs with long orbital periods. However, the mass transfer rate in BH SXTs is larger than in NS SXTs at short orbital periods (cf. eq. [5] and its scaling with $m_{1}$ ); the two are in fact quite close in Eddington units at these periods, where the major-

\footnotetext{
${ }^{7}$ See Verbunt \& van den Heuvel (1995) for a semianalytical model that allows one to compute tracks.
} 


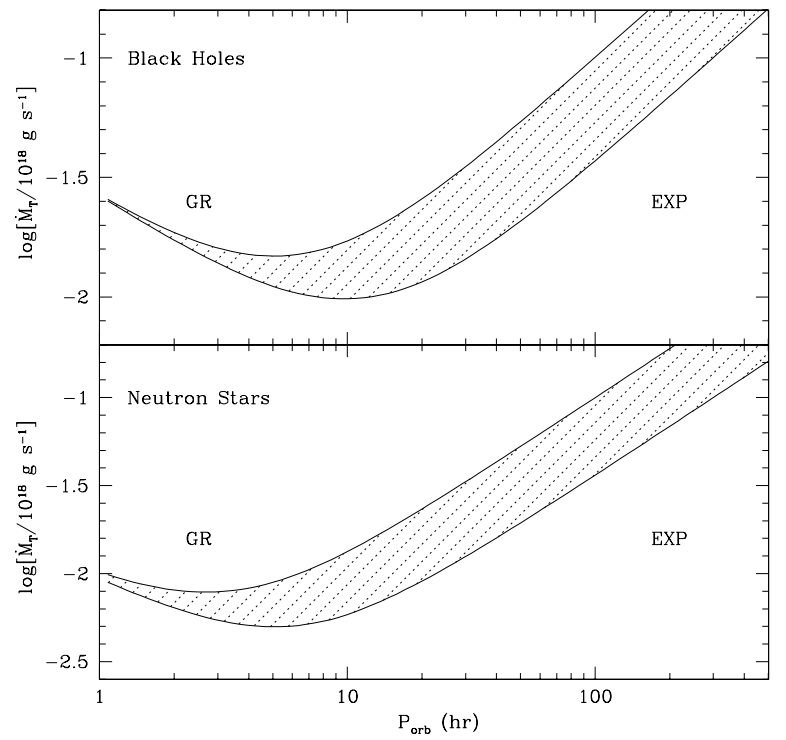

FIG. 3.-Predictions of binary-evolution models for the mass transfer rate $\dot{M}_{T}$ (in units of $10^{18} \mathrm{~g} \mathrm{~s}^{-1}$ ) in NS and BH SXTs, as a function of the orbital period $P_{\text {orb }}$. "GR" refers to a phase of mass transfer driven by gravitational radiation and "EXP" to mass transfer driven by secondary expansion.

ity of SXTs are found. Although this result neglects any contribution from magnetic braking, we think that it is significant enough to motivate the use of Eddington-scaled luminosities in Figures 1 and 2. Note that the comparable values of Eddington-scaled $\dot{m}_{T}$ predicted for BH SXTs and NS SXTs at short periods contrast with the large Eddington-scaled luminosity difference of the two classes of objects (Figs. 1 and 2). This in essence is the argument of Narayan et al. (1997b) and Garcia et al. (1998), and we confirm their claim. The sample is, however, small.

Although the estimates shown in Figure 3 rely on simple considerations that do not take into account the full complexity of mass transfer (in particular, substantial fluctuations of the mass transfer rates around the secular values used here may occur; see, e.g., Warner 1995), they probably provide better than an order-of-magnitude precision, which is sufficient for our discussion. Note, however, that this simple model is not likely to give an accurate value of $\dot{M}_{T}$ for the BH SXT J1655-40 since this system seems to be in a special evolutionary state (crossing the Hertzsprung gap; see, e.g., Kolb 1998).

\section{ACCRETION MODELS}

Following Narayan et al. (1996, 1997a), we assume that the accretion flow is made of two components: an inner ADAF and an outer thin accretion disk. Since only the ADAF contributes to the emission of quiescent SXTs in the $0.5-10 \mathrm{keV}$ band, we ignore the thin disk. Further, since the emission of the ADAF is primarily from regions close to the center, we do not concern ourselves with the precise value of the radius $R_{\text {tr }}$ at which the transition between the ADAF and the thin disk occurs.

Although the ADAF model was initially proposed for quiescent BH SXTs, there is no reason why it should not apply also to quiescent NS SXTs. In particular, if the transition of the accretion flow from a thin disk configuration to an ADAF configuration is triggered by the same local physics in the disk, e.g., a thermal instability in the upper layers of the disk (Shaviv \& Wehrse 1986; Meyer \& MeyerHofmeister 1994; Narayan \& Yi 1995b), the process should occur independently of the nature of the central object. We note, however, that the presence of a reradiating surface in the case of an NS primary at the center of the ADAF may have significant effects on the ADAF. For instance, Narayan \& Yi (1995b) showed that the critical accretion rate $\dot{m}_{\text {crit }}$ in an ADAF is reduced in the case of an NS (compared to a $\mathrm{BH}$ ) because the additional supply of soft photons (from the stellar surface) causes the ADAF to cool more efficiently. This is not an issue here since $\dot{m}$ in all the quiescent systems we study is well below $\dot{m}_{\text {crit }}$.

We assume conservative mass transfer. Consequently, if $\dot{M}_{\mathrm{ADAF}}$ is the rate at which mass is accreted during quiescence via the ADAF and $\dot{M}_{\text {accum }}$ is the rate at which mass is accumulated in the outer thin disk, the mass transfer rate satisfies $\dot{M}_{T}=\dot{M}_{\mathrm{ADAF}}+\dot{M}_{\text {accum }}$. The proportion of mass accreted versus mass accumulated in quiescence is, however, uncertain. Menou et al. (1999) estimated the relative importance of $\dot{M}_{\mathrm{ADAF}}$ and $\dot{M}_{\text {accum }}$ for most of the $\mathrm{BH}$ SXTs listed in Table 1 . They estimated $\dot{M}_{\text {accum }}$ by taking the integrated fluences of the outbursts of each source and assuming a radiative efficiency of $\eta=0.1$ (which provides the total mass accreted in the outburst) and estimated $\dot{M}_{\mathrm{ADAF}}$ by fitting the quiescent emission with an ADAF model. Menou et al.'s work indicates that, in quiescent BH SXTs, roughly one-half the matter that is transferred from the secondary is accreted via the ADAF and the rest is accumulated in the thin disk, i.e.,

$$
\frac{\dot{M}_{\mathrm{ADAF}}}{\dot{M}_{\mathrm{accum}}} \equiv g \sim 1, \quad \frac{\dot{M}_{\mathrm{ADAF}}}{\dot{M}_{T}}=\frac{g}{1+g} \sim \frac{1}{2} .
$$

In the following models, we treat $g$ as a free parameter, but we expect $g$ to be of order unity both in BH SXTs and in NS SXTs.

The version of the ADAF model used here is described in detail by Narayan et al. (1998). It consistently includes adiabatic compressive heating of electrons in the energy equation (Nakamura et al. 1997), and it uses for the flow dynamics the general relativistic global solutions calculated by Popham \& Gammie (1998).

In previous studies involving ADAFs, most of the model parameters were kept fixed at the following values (see Narayan et al. 1999 for a discussion): $\alpha_{\mathrm{ADAF}}=0.3$ (the viscosity parameter in the ADAF), $\beta \equiv P_{\text {gas }} / P_{\text {total }}=0.5$ (i.e., equipartition between the gas and the magnetic field), $\gamma=13 / 9=1.44$ (the adiabatic index of the gas), and $\delta=10^{-3}$ (the fraction of the viscous dissipation that goes directly into heating the electrons). We adopt these standard values of $\alpha_{\mathrm{ADAF}}, \beta$, and $\gamma$ in the present models (see $\S 6$ for the effect of varying $\delta$ ). We take $\dot{M}_{T}$ to be given by the predictions of binary-evolution models (Fig. 3) and obtain $\dot{M}_{\mathrm{ADAF}}$ via equation (6) for a given assumed value of $g$. We calculate the spectral energy distributions of the models and numerically integrate the spectra to obtain the luminosities in the $0.5-10 \mathrm{keV}$ band. The results are discussed in the following sections.

\subsection{ADAF Models of BH SXTs}

Figure $4 a$ shows two bands of luminosities corresponding to ADAF models of BH SXTs. In the upper band $\dot{M}_{\mathrm{ADAF}}=$ $\dot{M}_{T}$; i.e., the entire $\dot{M}_{T}$ is accreted by the black hole via the ADAF, while in the lower band $\dot{M}_{\mathrm{ADAF}}=\dot{M}_{T} / 3$ (or 

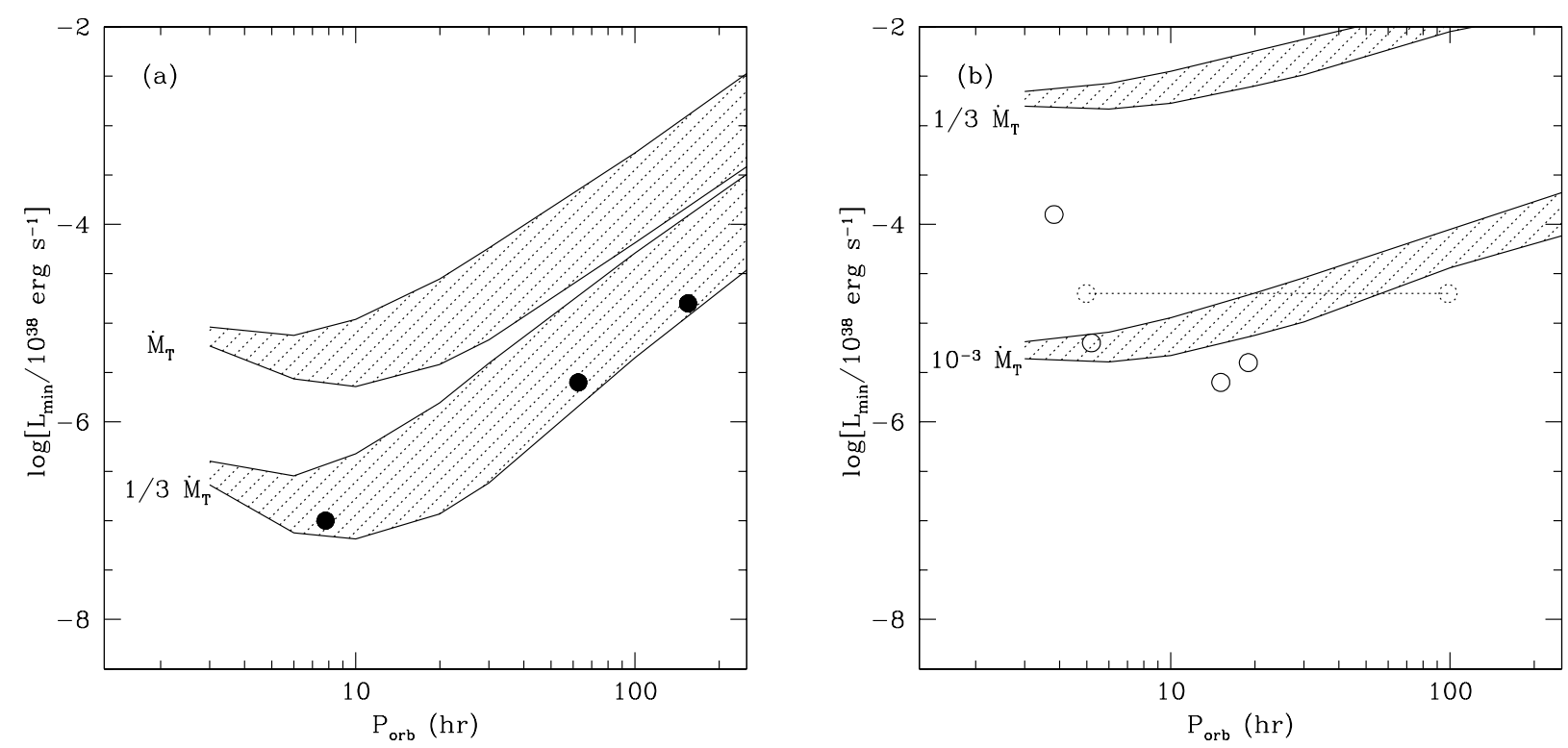

FIG. 4. - (a) Upper band shows the quiescent luminosities of BH SXTs in the $0.5-10 \mathrm{keV}$ band predicted by ADAF models if all the mass transferred by the secondary is accreted via the ADAF. The lower band corresponds to $\sim 1 / 3$ of the mass transferred being accreted via the ADAF. This model fits the observed luminosities reasonably well. Both bands have been calculated for $\alpha_{\mathrm{ADAF}}=0.3$. The effect of varying $\alpha_{\mathrm{ADAF}}$ is explained in the text. (b) Upper band shows the quiescent luminosities of NS SXTs predicted in the $0.5-10 \mathrm{keV}$ band if $1 / 3$ of the mass transferred by the secondary reaches the NS surface. The lower band shows that the luminosities actually observed correspond to a very small fraction $\left(\sim 10^{-3}\right)$ of the transferred mass reaching the NS surface.

$g=1 / 2$ ). The lower band is in satisfying agreement with the observed luminosities. The fact that we obtain agreement with a reasonable value of $g$ means that the modeling of the quiescent X-ray emission of $\mathrm{BH}$ SXTs with the ADAF model is consistent with the predictions of binary-evolution models. The increase in the luminosity with increasing $P_{\text {orb }}$ is also in good agreement. One should stress again, however, that the agreement for the BH SXT J1655-40 is unexpected if the system is crossing the Hertzsprung gap (Kolb 1998).

The bolometric radiative efficiency in the ADAF models constructed here is typically $\sim 10^{-3}$ compared to $\sim 10^{-1}$ for standard thin-disk accretion. The X-ray radiative efficiency of these ADAF models is another 2 or 3 orders of magnitude lower since much of the ADAF luminosity comes out as synchrotron radiation in the optical. Note that these results are sensitive to various details of the ADAF models, and in particular to the choice of $\alpha_{\mathrm{ADAF}}$ and $\beta$. For instance, we find that, if $\alpha_{\mathrm{ADAF}}=0.1$, we need $g \sim 1 / 4$ (i.e., a fraction $\sim 1 / 5$ of the mass supplied by the secondary must be accreted via the ADAF) in order to obtain agreement with the observed quiescent luminosities of BH SXTs. The fraction goes down further to $\sim 1 / 10$ if $\alpha_{\mathrm{ADAF}}=0.025$.

\subsection{ADAF Models of NS SXTS}

The presence of a hard surface on the accreting star is the key feature of ADAF models of NS SXTs. The large amount of energy advected in the ADAF, which is lost through the event horizon in BH SXTs, will be reradiated from the NS surface (Narayan \& Yi 1995b). Narayan et al. (1997b) and Garcia et al. (1998) argued that this additional source of luminosity is the explanation for the systematically larger luminosity of quiescent NS SXTs as compared to quiescent BH SXTs (Figs. 1 and 2). We now investigate this quantitatively.

In the following, we assume that the thermal energy stored in the ADAF is reradiated as blackbody emission with a radiative efficiency $\eta \sim 0.1$. The luminosity in the $0.5-10 \mathrm{keV}$ band will be dominated by the emission coming from the NS surface, so that the additional emission from the ADAF can be ignored (for simplicity). We also assume that the reradiation occurs from a small fraction $f_{\text {surf }}$ of the NS. This is in agreement with observations of quiescent NS SXTs (see, e.g., Verbunt et al. 1994; Campana et al. 1998; but see Rutledge et al. 1999 and the discussion in $\S 7$ ). For now, we do not seek to justify the small emitting area of the NS. We simply assume for all our NS models that the area is $10 \mathrm{~km}^{2}$. However, our results are not seriously affected even if the radiating area is increased or decreased by, say, an order of magnitude (i.e., as long as most of the blackbody emission is in the $0.5-10 \mathrm{keV}$ X-ray band). The typical effective temperatures found are $T_{\text {eff }} \lesssim 0.5 \mathrm{keV}$.

The upper band in Figure $4 b$ shows the luminosities predicted by binary-evolution models if $g=1 / 2$, i.e., one-third of the mass transferred by the secondary is accreted onto the NS (as in BH SXTs). We see that the luminosities predicted are much larger than those observed, by $\sim 3$ orders of magnitude (except for the system EXO 0748-676; see $\S 7$ for a discussion of this system). Since any contribution to the mass transfer by magnetic braking would only increase the mass transfer rates expected (in short orbital period systems), the discrepancy between the observed and predicted luminosities would be even more serious if magnetic braking were effective.

One possibility for the discrepancy is that most of the quiescent flux from NS SXTs is emitted outside the 0.5-10 $\mathrm{keV} \mathrm{X}$-ray band. This explanation seems, however, rather unlikely since this emission would be seen somewhere else in the spectrum, say in hard X-rays or soft $\gamma$-rays. Aq1 X-1 has been observed in quiescence in hard X-rays, and it is clear that the energy does not come out in the $10-100 \mathrm{keV}$ band (Campana et al. 1998). In addition, although some theoretical models of boundary layers between an accretion flow and an NS predict substantial deviations from black- 
body emission at higher energies (see, e.g., Shapiro \& Salpeter 1975; Turolla et al. 1994), there are no models predicting that such a large fraction $(\sim 0.999)$ of the emission would come out in hard X-rays or $\gamma$-rays.

We must therefore consider the possibility that only part of the mass supplied by the secondary actually reaches the surface of the NS. The lower band in Figure $4 b$ shows that the luminosities of quiescent NS SXTs can be accounted for (except EXO $0748-676$ ) if only $\sim 0.1 \%$ of the transferred mass reaches the NS surface. This is of course a surprisingly small fraction. ${ }^{8}$ In the following section, we argue that the propeller effect, if combined with accretion via an ADAF, can explain the fraction, while in $\S 6$ we consider the possibility that winds from ADAFs could further reduce the amount of mass reaching the NS surface.

\section{PROPELLER EFFECT IN QUIESCENT NEUTRON STAR SXTS}

The propeller effect in accreting neutron stars was initially proposed by Illarionov \& Sunyaev (1975) to explain the existence of long-period X-ray pulsars, and it was later developed in more detail by Davies \& Pringle (1981; see also Wang \& Robertson 1985; Stella, White, \& Rosner 1986). Although the details vary, the basic mechanism of the effect relies on the presence of a centrifugal barrier at the rotating magnetosphere of a rapidly spinning NS.

Inside the magnetosphere, the accreting gas is forced to follow the magnetic field lines of the NS since, by definition, in this region magnetic forces dominate the flow dynamics. The fate of the gas is then determined by the relative magnitudes of the magnetospheric radius, $R_{m}$, and the corotation radius, $R_{\mathrm{co}}$, defined by $\Omega_{\star} \equiv\left(G M / R_{\mathrm{co}}^{3}\right)^{1 / 2}$, where $\Omega_{\star}$ is the angular velocity of the NS (and the magnetosphere). If $R_{\mathrm{co}}<R_{m}$, the magnetosphere rotates so fast that the centrifugal force at $R_{m}$ is larger than the force of gravity and hardly any of the gas can be accreted onto the NS. This is the propeller effect. The efficiency of the propeller effect is not well understood (see Davies, Fabian, \& Pringle 1979; Davies \& Pringle 1981). It is also not clear whether the accreting matter is ejected from the system or is merely accumulated in a boundary layer around the magnetosphere (Wang \& Robertson 1985). However, from the simple physical argument outlined above, it is clear that normal accretion onto the neutron star is inhibited during the propeller phase.

The first direct observational evidence for the existence of the propeller effect in accreting neutron stars was reported by Cui (1997) in two X-ray pulsars, GX $1+4$ and GRO J1744-28. Cui showed that in both systems X-ray pulsations ceased during a period of low X-ray flux, which he interpreted as the result of a decrease in the mass accretion rate and a corresponding increase in the magnetospheric radius to beyond the corotation radius. Campana et al. (1998) and Zhang, Yu, \& Zhang (1998) argued that the propeller effect was also seen in an NS SXT, Aql X-1, during its most recent outburst; the system showed an abrupt decrease of the X-ray flux, accompanied by considerable hardening of the X-ray spectrum. Both sets of authors pro-

\footnotetext{
${ }^{8}$ Although this value might simply be interpreted, in a scenario without ADAF, as the accretion rate at the inner edge of an unsteady quiescent disk $\left(\dot{M} \propto R^{2.5-3}\right.$ in such a disk; see, e.g., Cannizzo 1993), we do not favor this interpretation because the propeller effect should operate in this case and prevent any mass from reaching the NS surface (see $\S 5$ ).
}

posed that the hard spectrum originates just outside the magnetosphere, where the gas becomes very hot because of the action of the propeller (see also Cui et al. 1998).

Observations of Aq1 X-1 show that even in quiescence the blackbody spectral component does not disappear entirely, implying that a small amount of material continues to accrete onto the star, contrary to simple models of the propeller (Verbunt et al. 1994; Stella et al. 1994). To resolve this problem, Zhang et al. (1998) proposed that accretion in quiescent NS SXTs occurs via a quasi-spherical ADAF, rather than a thin disk. This allows some material to accrete near the poles, thereby bypassing the centrifugal barrier. In this section we develop this idea quantitatively.

\subsection{Accretion Geometry in Quiescence}

In the following, we assume for simplicity that the NS spin axis is perpendicular to the binary orbital plane and that the NS spin and magnetic axes are aligned.

\subsubsection{Magnetospheric Radius}

In the presence of a spherical accretion flow (see, e.g., Bondi 1952), the magnetospheric (or Alfvén) radius, $R_{m}$, is usually defined as the radius at which the magnetic pressure, $P_{\text {mag }}=B^{2} / 8 \pi$, due to the neutron star magnetic field balances the ram pressure, $P_{\text {ram }} \equiv \rho v_{r}^{2} / 2$, of the accreting gas, where $v_{r}$ is the radial infall velocity of the gas (see, e.g., Frank et al. 1992). The contribution from the thermal pressure of the gas is generally neglected when considering Bondi-type flows, which are highly supersonic (free falling). An ADAF, on the other hand, is subsonic down to a few Schwarzschild radii (see, e.g., Narayan, Kato, \& Honma 1997c; Chen, Abramowicz, \& Lasota 1997b; Abramowicz et al. 1996), and the thermal pressure term must be taken into account.

The polar structure of ADAFs was described by Narayan \& Yi (1995a) in the self-similar approximation. They showed that in the limit of strong advection $\left(f_{\text {adv }} \sim 1\right)$, the gas density and temperature (and therefore the thermal pressure of the gas) are essentially independent of the polar angle $\theta$; i.e., these quantities are nearly constant on spherical shells centered on the accreting object. On the other hand, the radial infall velocity is smaller than the thermal sound speed in the equatorial plane and decreases toward the poles. Clearly then, the thermal pressure of the accreting material is the dominant term in calculating the magnetospheric radius. Since this pressure is constant with $\theta$, the magnetosphere will be nearly spherical.

Despite the difference between a Bondi spherical accretion flow and an ADAF, the value of $R_{m}$ is roughly the same in the two cases. In the former, essentially all the gravitational energy released during accretion goes into bulk kinetic energy of the flow, while in ADAFs, the energy is stored as thermal energy of the gas. Thus, the ram pressure in Bondi flows must be of the same order as the thermal pressure in ADAFs. The magnetospheric radius in quiescence then takes the same form (Frank et al. 1992):

$$
R_{\mathrm{mq}}=6.45 \times 10^{5}\left(\frac{\dot{M}}{\dot{M}_{\mathrm{Edd}}}\right)^{-2 / 7} m_{1}^{-3 / 7} B_{8}^{4 / 7} R_{\mathrm{NS}, 6}^{12 / 7} \mathrm{~cm},
$$

where $B_{8}$ is the NS surface magnetic field in units of $10^{8} \mathrm{G}$ and $R_{\mathrm{NS}, 6}$ is the NS radius in units of $10^{6} \mathrm{~cm}$.

For the remainder of the paper, $R$ refers to the radius in physical units and $r$ refers to the radius in Schwarzschild units. 


\subsubsection{Accretion in the Propeller Phase}

The propeller effect in binary systems is generally discussed in the context of thin-disk accretion. In this model, once the propeller becomes effective, no matter is able to reach the NS surface because the centrifugal acceleration acts equally against gravity for all the matter located in the disk orbital plane.

In a spherical flow, however, the centrifugal acceleration at the magnetospheric radius acting on a parcel of gas, accreting at a polar angle $\theta$ from the spin axis, is equal to $A_{c}=\Omega_{\star}^{2} R_{\mathrm{mq}} \sin \theta$, where $\Omega_{\star}$ is the angular speed of the magnetic field lines anchored in the NS. The direction of this force is perpendicular to the spin axis of the NS (and parallel to the orbital plane), so that the component of $A_{c}$ along the radial direction is simply $A_{c} \sin \theta=$ $\Omega_{\star}^{2} R_{\mathrm{mq}} \sin ^{2} \theta$. The maximum polar angle below which the gravitational acceleration $\left(=R_{\mathrm{mq}} \Omega_{K}^{2}\left(R_{\mathrm{mq}}\right)\right)$ wins over the centrifugal force is given by

$$
\sin \theta_{0}=\frac{\Omega_{K}\left(R_{\mathrm{mq}}\right)}{\Omega_{\star}}
$$

For $\theta_{0} \ll 1$ this simplifies to

$$
\theta_{0} \simeq \frac{\Omega_{K}\left(R_{\mathrm{mq}}\right)}{\Omega_{\star}}
$$

Note that the residual force acting on all parcels of gas, even those that do not overcome the centrifugal barrier, tends to direct them toward the orbital plane. In this simple formulation, we do not consider possible complications due to field-line orientation effects.

\subsubsection{Fraction of Mass Reaching the NS Surface}

Only matter accreting between $\theta=0$ and $\theta=\theta_{0}$ can overcome the centrifugal barrier and reach the surface of the NS. The mass accretion rate onto the star is then

$$
\dot{M}_{\mathrm{NS}}=-2 \int_{0}^{\theta_{0}} 2 \pi R \sin \theta \rho v_{r} R d \theta,
$$

where $\rho=\rho(R, \theta) \simeq \rho(R)$ is the gas density and $v_{r}=$ $v_{r}(R, \theta) \simeq v_{r}(R) \sin ^{2} \theta$ is the radial infall velocity at angle $\theta$ (positive outward), as given by the self-similar solution of Narayan \& Yi (1995a). The factor of 2 in equation (10) is introduced to account for the two polar caps. The fraction of the total mass accretion rate $\dot{M}_{\mathrm{ADAF}}$ in the ADAF that reaches the NS surface is then

$$
\begin{aligned}
f_{\mathrm{acc}} & \equiv \frac{\dot{M}_{\mathrm{NS}}}{\dot{M}_{\mathrm{ADAF}}} \simeq \frac{2 \int_{0}^{\theta_{0}} 2 \pi R^{2} \sin \theta \rho(R) v_{r}(R) \sin ^{2} \theta d \theta}{2 \int_{0}^{\pi / 2} 2 \pi R^{2} \sin \theta \rho(R) v_{r}(R) \sin ^{2} \theta d \theta} \\
& =\frac{2 \int_{0}^{\theta_{0}} \sin ^{3} \theta d \theta}{2 \int_{0}^{\pi / 2} \sin ^{3} \theta d \theta} \\
& \simeq \frac{3}{8} \theta_{0}^{4} \simeq \frac{3}{8}\left[\frac{\Omega_{K}\left(R_{\mathrm{mq}}\right)}{\Omega_{\star}}\right]^{4},
\end{aligned}
$$

where the last two steps have been calculated in the limit of small $\theta_{0}$. For a neutron star with a spin period of $2 \mathrm{~ms}$, with a magnetic field strength of $3 \times 10^{8} \mathrm{G}$, and accreting at $\dot{M}_{\text {ADAF }}=10^{-3} \dot{M}_{\text {Edd }}$, we find $f_{\text {acc }} \simeq 0.75 \times 10^{-3}$. Note that if the radial velocity does not vary as $\sin ^{2} \theta$ but is more nearly constant with $\theta$ (as in Bondi accretion), then $f_{\text {acc }}$ would be larger [e.g., if $v_{r}(R, \theta)$ is independent of $\theta, f_{\text {acc }} \propto \theta_{0}^{2}$ only]. On the other hand, if the accretion flow has a toroidal morphology with empty funnels along the rotation axis, $f_{\text {acc }}$ would be much lower than the expression given in equation (11), perhaps even zero. There is thus considerable uncertainty in the value of $f_{\text {acc }}$.

\subsubsection{Fraction of the NS Surface Emitting Radiation}

We assume that the gas accreted during the propeller phase follows the magnetic field lines down to the NS polar caps, where its kinetic and thermal energy is converted to radiation. Dipolar field lines satisfy the parametric equation (see, e.g., Frank et al. 1992):

$$
R=C \sin ^{2} \theta,
$$

where $C$ is constant for a given field line. Thus, the field lines intersecting the magnetospheric radius at $\theta=\theta_{0}$ emerge from the NS surface at a polar angle $\theta_{S}$ given by

$$
\frac{\sin ^{2} \theta_{S}}{\sin ^{2} \theta_{0}}=\frac{R_{\mathrm{NS}}}{R_{\mathrm{mq}}} .
$$

The accreting material reaches the NS surface at $\theta \leq \theta_{S}$, and therefore the fraction of the neutron star surface that reradiates the accreted energy is

$$
f_{\text {surf }} \equiv \frac{2 \int_{0}^{\theta_{S}} 2 \pi \sin \theta d \theta}{2 \int_{0}^{\pi / 2} 2 \pi \sin \theta d \theta} \simeq \frac{\theta_{S}^{2}}{2} \simeq \frac{\theta_{0}^{2}}{2} \frac{R_{\mathrm{NS}}}{R_{\mathrm{mq}}},
$$

where we have made use of the fact that $\theta_{S}<\theta_{0} \ll 1$. Note the direct dependence of $f_{\text {surf }}$ on the NS magnetic field strength $B$ through $R_{\mathrm{mq}}$. For a neutron star with a spin period of $2 \mathrm{~ms}$, with a magnetic field strength of $3 \times 10^{8} \mathrm{G}$, and accreting at $\dot{M}_{\mathrm{ADAF}}=10^{-3} \dot{M}_{\mathrm{Edd}}$, we find $f_{\text {surf }} \simeq 3$ $\times 10^{-3}$.

\subsection{Equilibrium Spin Frequency}

Equations (11) and (14) demonstrate that in quiescence the mass accretion rate onto an NS and the emitting area, which determine the total luminosity and spectrum of the system, depend strongly on the NS angular rotation speed $\Omega_{\star}$ and the field strength. Although $\Omega_{\star}$ is not known for most NS SXTs, we discuss in this section how it can be estimated using our knowledge of the accretion history of an SXT and of the interaction between the magnetosphere of the NS and the accretion flow.

During the propeller phase, in quiescence, the NS ejects most of the accreting gas that reaches its magnetosphere. The ejected material leaves the system with higher specific angular momentum than it had coming in, which results in an effective spin-down of the NS. However, NS SXTs experience outbursts during which the mass accretion rate onto the NS is orders of magnitude higher than in quiescence. High $\dot{M}$ causes the magnetosphere to shrink inside the corotation radius so that all the accreting gas is able to reach the NS surface. The mass and angular momentum of the accreting gas is added to the NS, which causes it to spin up. An NS SXT that accretes for a fairly long time is expected to reach an equilibrium spin frequency $\Omega_{\star \text {,eq }}$ such that the spin-up during outburst is balanced by the spindown during quiescence.

At the onset of mass transfer, the spin period of the NS in an SXT is likely to be different from the equilibrium value. In standard Ghosh \& Lamb (1979)-type models (for steady disks), the timescale $\tau_{\text {eq }}$ on which a typical NS reaches the equilibrium spin period is usually small ( $\left.\gtrsim 10^{5} \mathrm{yr}\right)$ compared to the lifetime of the low-mass X-ray binary (LMXB; $10^{8}-10^{9} \mathrm{yr}$; see, e.g., Henrichs 1983 for a review). Although 
$\tau_{\text {eq }}$ may be a little larger for transient accretion, it is reasonable to assume that most of the neutron stars in SXTs have rotation rates close to $\Omega_{\star \text {,eq }}$. It is straightforward to show that $\Omega_{\star}$ is basically unaffected by a single outburst or a single quiescent phase, because the time spent between two outbursts (about a few years) is much shorter than $\tau_{\text {eq }}$. Therefore, it is not necessary to consider the small jitter in $\Omega_{\star}$ during the quiescence-outburst cycle. In the following, we simply assume that once an NS SXT has reached spin equilibrium, then $\Omega_{\star}=\Omega_{\star \text {,eq }}$ both during outburst and quiescence.

\subsubsection{Idealized Model for the Spin-up and Spin-down Torques}

To determine the equilibrium spin frequency of an NS in a transient binary system, we need a theoretical description of the interaction between the NS and the accretion flow. We begin with the outburst phase during which accretion occurs via a thin disk and the torque exerted by the accreting gas on the NS is relatively well understood.

Despite some modifications, current models describing the interaction between an NS and a magnetically threaded thin accretion disk remain essentially identical to the model initially proposed by Ghosh \& Lamb (1979). In this model, the spin-up torque on the NS results from two contributions. First, the gas that follows magnetic field lines and reaches the NS surface gives its angular momentum to the NS (spin-up). Second, the interaction between the magnetic field lines and the threaded thin disk beyond the magnetospheric radius results in a positive torque on the NS for radii where the field lines rotate more slowly than the local Keplerian angular speed of the gas. The lines that thread the disk farther out give a (smaller) negative torque on the NS since these field lines rotate more quickly than the local Keplerian angular speed of the gas (Ghosh \& Lamb 1979). Recent detailed numerical simulations by Daumerie (1996) show that the overall contribution resulting from the interaction of the accretion flow and the magnetic field lines beyond the magnetospheric radius is nearly equal to the contribution to the spin-up from the gas reaching the NS surface. Further, Daumerie finds that the torque varies roughly linearly with the fastness parameter $\omega \equiv$ $\Omega_{\star} / \Omega_{K}\left(R_{m}\right)$.

Using this work as a guide, we propose the following idealized formula for the torque:

$$
\dot{J}=2 \dot{M} R_{m}^{2} \Omega_{K}\left(R_{m}\right)\left\{1-\left[\frac{\Omega_{\star}}{\Omega_{K}\left(R_{m}\right)}\right]\right\},
$$

where the factor of 2 appears because of the two nearly equal contributions to the spin-up mentioned above. Equation (15) assumes that the fastness parameter at equilibrium, $\omega_{\text {crit }}$, is exactly unity. This is consistent with the recent arguments of Wang (1995; see also Wang 1987).

The torque in the propeller regime, when $\Omega_{\star}>\Omega_{K}\left(R_{m}\right)$, is not as well constrained. By analogy with Ghosh \& Lambtype models, we assume that the torque arises from two nearly equal contributions. One contribution is the negative torque applied to the NS when the propeller expels gas at the magnetosphere, and the other is the negative torque ${ }^{9}$ due to the interaction of the magnetic field lines with the accretion flow beyond the magnetospheric radius. If the gas expelled leaves the magnetosphere with an angular momen-

\footnotetext{
${ }^{9}$ This contribution to the torque is always negative in the propeller regime because $\Omega_{\star}>\Omega_{K}(R)$ for all $R>R_{\mathrm{mq}}$.
}

tum corresponding to the angular rotation speed of the NS, viz., $\Omega_{\star}$ (which corresponds to an efficient propeller), then the negative torque on the NS in the propeller phase is

$$
\dot{J} \sim-2 \dot{M} R_{m}^{2} \Omega_{\star},
$$

where $\dot{M}$ is the rate at which mass reaches the magnetosphere. (The small positive contribution from the very small fraction of mass accreted on to the NS is neglected.) This expression is in agreement with equation (15) in the limit of $\Omega_{\star} \gg \Omega_{K}\left(R_{m}\right)$. Therefore, we can use the prescription given by equation (15) to describe the interaction between the NS and the accretion flow throughout the outburst-quiescence cycle.

\subsubsection{Spin Equilibrium}

We define $\Delta J_{o}=\dot{J}_{o} \Delta t_{o}$ to be the integrated spin-up torque over an outburst of duration $\Delta t_{o}$ and $\Delta J_{q}=\dot{J}_{q} \Delta t_{q}$ to be the integrated spin-down torque during a propeller phase of duration $\Delta t_{q}$. The recurrence time of the SXT is $\Delta t_{o}+\Delta t_{q}$. We assume, for simplicity, that the two phases can be approximated as bimodal, with the outburst phase occurring at a typical accretion rate $\dot{M}_{o}$ and the quiescent phase having a typical lower accretion rate $\dot{M}_{q}$. These accretion rates define, in turn, the values of the magnetospheric radius in outburst, ${ }^{10} R_{\mathrm{mo}}$, and in quiescence, $R_{\mathrm{mq}}$, used in equation (15) to determine $\dot{J}_{o}$ and $\dot{J}_{q}$. Since the accretion rate from the peak of the outburst is known to decrease gradually with time, the value of $\dot{M}_{o}$ used to determine $R_{\text {mo }}$ should be interpreted as a mean over the outburst.

By definition, the NS angular rotation speed at equilibrium $\Omega_{\star \text {,eq }}$ satisfies $\Delta J_{o}=\Delta J_{q}$. Using equation (15), we find

$$
\Omega_{\star, \mathrm{eq}}=\Omega_{K}\left(R_{\mathrm{mq}}\right)\left(\frac{R_{\mathrm{mq}}}{R_{\mathrm{mo}}}\right)^{3 / 2} \frac{\left[1+\left(R_{\mathrm{mq}} / R_{\mathrm{mo}}\right)^{1 / 2} \tilde{g}\right]}{\left[1+\left(R_{\mathrm{mq}} / R_{\mathrm{mo}}\right)^{2} \tilde{g}\right]},
$$

where $\tilde{g}$ is the ratio $\dot{M}_{q} \Delta t_{q} / \dot{M}_{o} \Delta t_{o}$, i.e., the total mass reaching the magnetosphere during the ADAF-propeller phase divided by the total mass accreted during outburst. This definition of $\tilde{g}$ is equivalent to the definition of $g$ given in equation (6) if all the mass accumulated in the disk during quiescence is accreted onto the neutron star during outburst. ${ }^{11}$ For the remainder of the paper, we assume $g=\tilde{g}$. Note that the spin equilibrium period defined by equation (17) differs from the spin equilibrium period defined for steady accretion onto an NS and usually discussed in the literature (e.g., Henrichs 1983).

\subsection{Propeller Regime at Spin Equilibrium}

Equation (17) allows us to determine the equilibrium spin period $P_{\text {spin }}$ as a function of the parameter $g=$ $\dot{M}_{\mathrm{ADAF}} / \dot{M}_{\text {accum }}$. This, in turn, allows us to estimate the quantities $f_{\text {acc }}$ (eq. [11]) and $f_{\text {surf }}$ (eq. [14]), which are relevant for determining the observational properties of quiesc-

\footnotetext{
${ }^{10}$ Following Frank et al. (1992), we use a value for the magnetospheric radius of the thin disk that is one-half that used for spherical accretion (eq. [7]).

${ }_{11}$ A possible situation in which the mass accreted onto the neutron star during outburst is less than the mass accumulated in the disk during quiescence is if the depletion rate of the disk during outburst is super Eddington while the accretion rate onto the neutron star is Eddingtonlimited. Even in that case, eq. (17) remains valid because $\Omega_{\star \text {, eq }}$ depends on the ratio $\dot{M}_{q} \Delta t_{q} / \dot{M}_{o} \Delta t_{o}$.
} 
ent NS SXTs. In the following calculations, we assume for definiteness that the NS accretes at the Eddington rate $\left(\dot{M}_{o}=\dot{M}_{\text {Edd }}\right)$ in outburst and that the mass transfer accretion rate in quiescence is $\dot{M}_{T}=10^{-2.5} \dot{M}_{\text {Edd }}$ (cf. Fig. 3). However, our conclusions do not depend crucially on these assumptions.

Figures 5 and 6 show the variations of $\dot{M}_{\mathrm{NS}} / \dot{M}_{T}=f_{\text {acc }}$ $\times g /(1+g), f_{\text {surf }}$, and $P_{\text {spin }}$ with $g$. As $\dot{M}_{\text {ADAF }}$ decreases, the magnetosphere becomes more extended and the propeller effect becomes more efficient. The solid lines show the results for an NS with a magnetic field strength $B=10^{8} \mathrm{G}$,

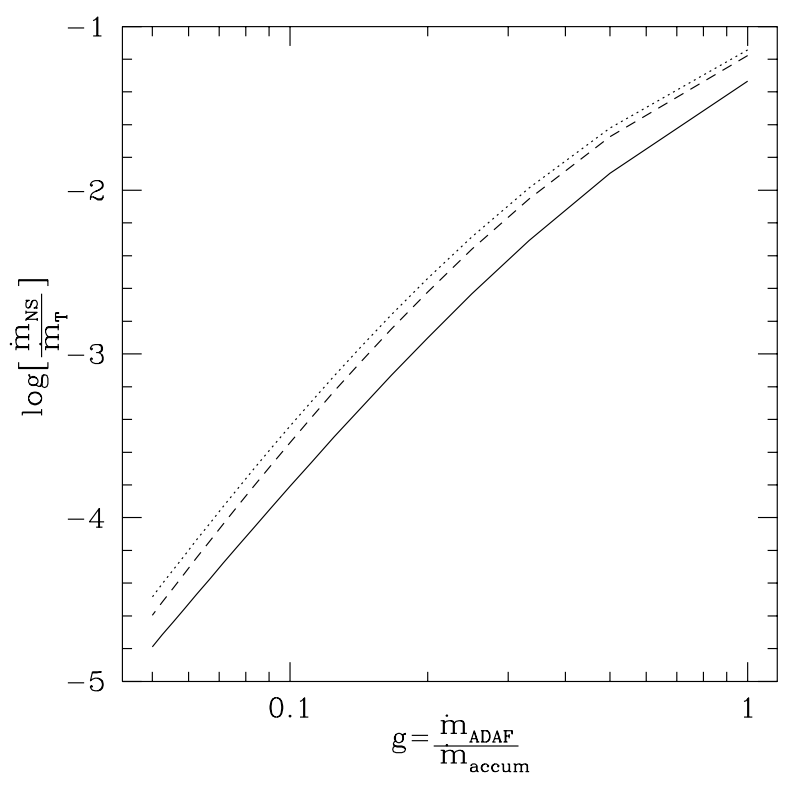

FIG. 5.-Fraction of mass transferred by the secondary that reaches the neutron star surface in the propeller regime, as a function of $g=$ $\dot{M}_{\mathrm{ADAF}} / \dot{M}_{\text {accum }}$. The solid line shows this fraction for a neutron star with a surface magnetic field strength of $10^{8} \mathrm{G}$, while the dashed and dotted lines correspond to $10^{9} \mathrm{G}$ and $10^{10} \mathrm{G}$, respectively.

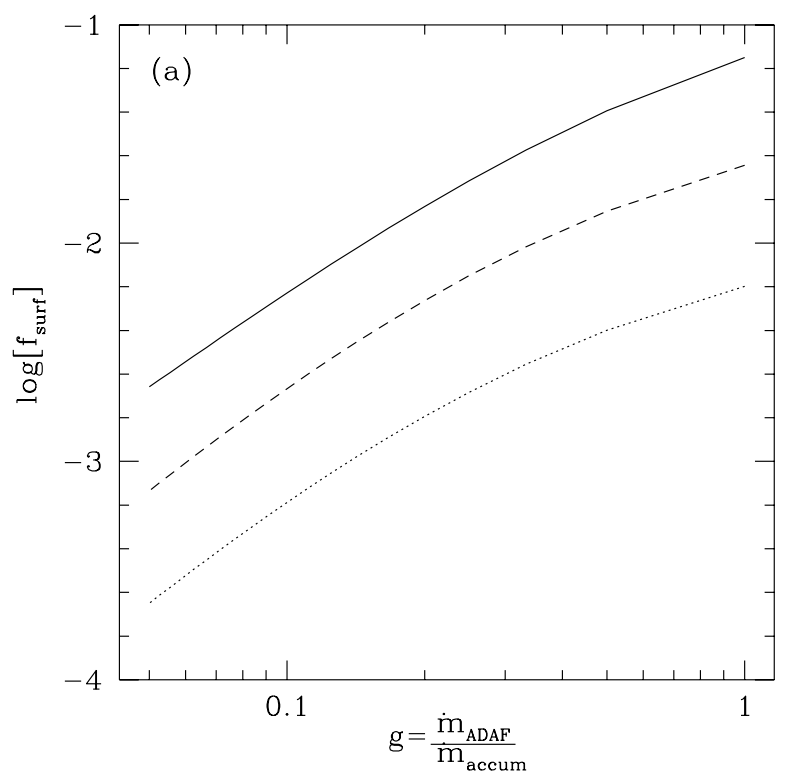

while the dashed and dotted lines show the cases $B=10^{9} \mathrm{G}$ and $B=10^{10} \mathrm{G}$, respectively. Recent observations suggest that accreting neutron stars in low-mass binaries have rather low magnetic fields, typically $B \lesssim 10^{9} \mathrm{G}$ (see, e.g., White \& Zhang 1997).

Figure 5 can be related to Figure $4 b$, where we found that $\dot{M}_{\mathrm{NS}} / \dot{M}_{T} \sim 10^{-3}$ was needed in order to explain the very low observed luminosities of quiescent NS SXTs. Such a value of $\dot{M}_{\mathrm{NS}} / \dot{M}_{T}$ is predicted by the propeller model if $g \sim 1 / 5$. This value of $g$ is reasonably close to the value $g \sim 1 / 2$ that we obtained for BH SXTs. Note that the dependence of $\dot{M}_{\mathrm{NS}} / \dot{M}_{T}$ on the magnetic field $B$ should cancel out through the ratios $R_{\mathrm{mq}} / R_{\mathrm{mo}}$ in equation (17). For low magnetic field strengths, however, the inner edge of the disk in outburst is not given by one-half the value in equation (7) but is fixed at the last stable orbit at 3 Schwarzschild radii (for instance, if $\dot{M}_{o} \geq 0.1 \dot{M}_{\mathrm{Edd}}$ and $B \leq 10^{9} \mathrm{G}$ ). Consequently, there is a residual dependence of $\dot{M}_{\mathrm{NS}} / \dot{M}_{T}$ on $B$ for small $B$.

Figure $6 a$ shows that the fraction $f_{\text {surf }}$ of the NS surface that emits in quiescence depends much more strongly on $B$ than $f_{\text {acc }}$ does (Fig. 5), as expected from equation (14). For values of $g \sim 1 / 5$ and low magnetic fields $\left(B \lesssim 10^{9} \mathrm{G}\right)$, the emitting surface is typically between 1 and $10 \mathrm{~km}^{2}$. Observations of quiescent NS SXTs suggest an emitting surface $\sim 1 \mathrm{~km}^{2}$ (see, e.g., Verbunt et al. 1994; Campana et al. 1998; but see $\S 7$ ). Given that the surface could very well be underestimated by the standard techniques (see, e.g., Lewin, van Paradijs \& Taam 1993; Rajagopal \& Romani 1996; Zavlin, Pavlov, \& Shibanov 1996; Rutledge et al. 1999), values of $\sim 1-10 \mathrm{~km}^{2}$ appear in reasonable agreement with the observations. We note, however, that this result depends quite crucially on the assumed alignment of the NS spin and magnetic axes. This is a major uncertainty in the calculations.

Figure $6 b$ shows that $P_{\text {spin }}$ is even more sensitive to the value of $B$. For weak magnetic fields $\left(B \lesssim 10^{9} \mathrm{G}\right)$, the values of the equilibrium spin period are in the range $1-10 \mathrm{~ms}$,

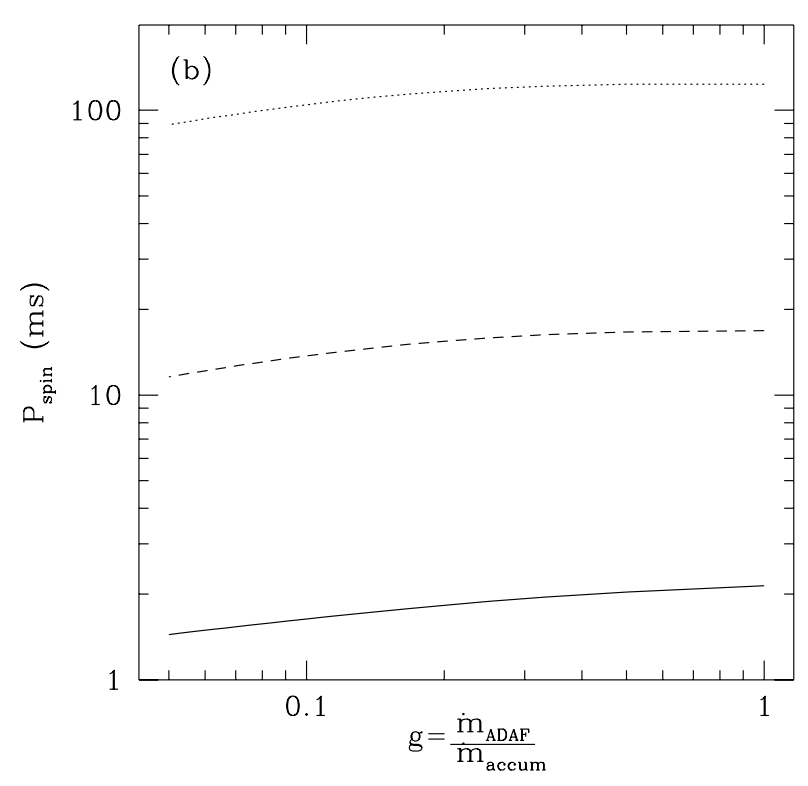

FIG. 6.-(a) Fraction $f_{\text {surf }}$ of the neutron star surface emitting radiation in the propeller regime, as a function of $g=\dot{M}_{\text {ADAF }} / \dot{M}_{\text {accum }}$. The solid line shows $f_{\text {surf }}$ for a neutron star with a surface magnetic field strength of $10^{8} \mathrm{G}$, while the dashed and dotted lines correspond to $10^{9} \mathrm{G}$ and $10^{10} \mathrm{G}$, respectively. (b) Equilibrium spin period $P_{\text {spin }}$ as a function of $g=\dot{M}_{\text {ADAF }} / \dot{M}_{\text {accum }}$ for the same magnetic field strengths as in $(a)$. 
which is in agreement with values recently discussed in the literature for accreting neutron stars in low-mass binaries (see, e.g., White \& Zhang 1997).

In our simplified model for the propeller, there are several sources of uncertainty, which are discussed in $\S \S 6.2$ and 7 below. The proximity of the value $g \sim 1 / 2$ required to explain the quiescent luminosities of BH SXTs to the value $g \sim 1 / 5$ required to explain the luminosities of NS SXTs (with propellers) suggests, however, that roughly the same geometry of accretion (outer thin disk and inner ADAF sharing the mass supplied by the secondary roughly equally) can account for the properties of both classes of objects. The presence of a hard surface and a magnetic field associated with the spinning neutron star are two major ingredients of the model. The NS SXTs Aq1 X-1 $\left(B \sim 10^{8} \mathrm{G}\right.$, $P_{\text {spin }} \sim 2-3 \mathrm{~ms}$, emitting area $\sim 1 \mathrm{~km}^{2}$; White \& Zhang 1997; Zhang et al. 1998; Campana et al. 1998) and SAX J1808.4-3658 $\left(B<2 \times 10^{8} \mathrm{G}, P_{\text {spin }}=2.49 \mathrm{~ms}\right.$; Wijnands \& van der Klis 1998; Chakrabarty \& Morgan 1998, hereafter CM98) are two interesting candidates that could allow further testing of the predictions of our ADAF-propeller scenario.

We note that CM98 inferred a mass transfer rate in SAX $\mathrm{J} 1808.4-3658 \approx 10^{-11} M_{\odot} \mathrm{yr}^{-1}$, which is roughly a factor of 10 less than the value for a $2 \mathrm{hr}$ orbital period NS SXT according to our Figure 3. These authors find that the estimate is consistent with mass transfer being driven by angular momentum losses due to gravitational radiation if the secondary star has a mass $m_{2}=0.05$. The values shown in Figure 3, however, were deduced assuming that the donor star is a main-sequence star that satisfies $m_{2}=$ $0.11\left(P_{\text {orb }} / 1 \mathrm{hr}\right)(\S 3)$. This is apparently not the case in SAX J1808.4-3658, perhaps because of the fact that the companion star is strongly irradiated (CM98).

\section{WINDS FROM ADAFS AND THE EFFICIENCY OF THE PROPELLER EFFECT}

The gas in an ADAF could in principle escape to infinity via a wind because part of the flow may be unbound. Narayan \& Yi $(1994,1995 a)$ showed that the Bernoulli constant, a measure of the energy of the gas at infinity, is positive in their self-similar solution, and Narayan et al. (1997c) showed that this is also the case in a global solution. A simple way of taking this into account was proposed by Blandford \& Begelman (1999, hereafter BB99), who derived self-similar ADAF solutions that include mass loss via winds.

Our results in $\S 4.1$ suggest that reducing $\alpha_{\mathrm{ADAF}}$ has only a small effect on the radiative efficiency of the accretion flow. Quataert \& Narayan (1999) showed, however, that an efficient way of increasing the radiative efficiency of the ADAF is to include winds and simultaneously increase the value of the parameter $\delta$, the fraction of the viscously dissipated energy that directly heats the electrons. They found that models with winds and high $\delta$ are consistent with the current observations for the BH SXT V404 Cyg in quiescence and the Galactic center source Sgr A*. Note that theoretical estimates of the value of $\delta$ are highly uncertain. Quataert (1998) and Gruzinov (1998) argued that $\delta$ could be small if the magnetic fields in the ADAF are fairly subthermal, but Bisnovatyi-Kogan \& Lovelace (1997; see also Quataert \& Gruzinov 1999) argued that magnetic reconnection in the accretion flow could preferentially heat the electrons and lead to large values of $\delta(\sim 1)$.
In $\S \S 6.1$ and 6.2, we investigate the following two questions. Can winds by themselves, i.e., without any propeller effect, explain the observed quiescent luminosities of NS SXTs? If not, is a combination of winds and a propeller consistent with the observations?

\subsection{ADAF Models of NS and BH SXTs with Winds}

Following BB99, we assume that the accretion rate in the ADAF scales in a self-similar way, i.e.,

$$
\dot{m}(r)=\dot{m}\left(r_{\mathrm{tr}}\right)\left(\frac{r}{r_{\mathrm{tr}}}\right)^{p}
$$

where $\dot{m}$ is the accretion rate scaled in Eddington units ( $\dot{m}=\dot{M} / \dot{M}_{\text {Edd }}$ ), $0<p<1$, and we assume that the wind is effective over the entire radial extent of the ADAF out to $r_{\text {out }}=r_{\text {tr }}$, the transition radius.

The efficiency of mass loss is determined not only by $p$, but also by the radial extent of the ADAF (eq. [18]). Around a black hole, the ADAF extends from the transition radius $\left(r_{\mathrm{tr}}\right)$ down to the event horizon, and so the accretion rate onto the black hole is $\dot{m}_{\text {in }}=\dot{m}\left(r_{\text {tr }}\right)\left(1 / r_{\text {tr }}\right)^{p}$. Around a neutron star, however, the ADAF has a minimum radius equal to the magnetospheric radius $r_{m}$, so that the accretion rate at the magnetosphere is $\dot{m}_{\mathrm{in}}=\dot{m}\left(r_{\mathrm{tr}}\right)\left(r_{m} / r_{\mathrm{tr}}\right)^{p}$. If $r_{m} \gg 1$, as is the case for $r_{\mathrm{mq}}$ in quiescent NS SXTs, then for a given value of $p$, the presence of a magnetosphere around the NS significantly reduces the total mass loss relative to the $\mathrm{BH}$ case. The value of $r_{m}$ itself depends on the local accretion rate at the magnetosphere (eq. [7]). Since the accretion rate at a given radius decreases with increasing $p$, a larger $p$ implies both a larger $r_{m}$ and a smaller radial extent for the ADAF.

We solve for the magnetospheric radius by combining equations (7) and (18) and find

$$
r_{\mathrm{mq}}=\left[1.35 \times \dot{m}\left(r_{\mathrm{tr}}\right)^{-2} r_{\mathrm{tr}}^{2 p} B_{8}^{4} R_{\mathrm{NS}, 6}^{12}\right]^{1 /(7+2 p)},
$$

where $r_{\mathrm{mq}}$ is expressed in Schwarzschild units for an NS of $1.4 M_{\odot}$ (eq. [19] is the generalization of eq. [7] for nonzero $p$ ). We have confirmed (by combining eqs. [18] and [19]) that, despite the reduction of the radial extent of the ADAF for larger values of $p$, increasing $p$ leads to an effective reduction of $\dot{m}\left(r_{\mathrm{mq}}\right)$ for any reasonable values of $p, r_{\mathrm{tr}}$ and $\dot{m}\left(r_{\text {tr }}\right)$.

For a standard NS with low magnetic field strength $\left(B_{8}=1, R_{\mathrm{NS}, 6}=1\right)$ and a typical accretion rate at the outer boundary of the ADAF $\dot{m}\left(r_{\mathrm{tr}}\right)=1 / 3 \times 10^{-2.5}(\S 4.2$; Fig. 3$)$, we find $r_{\mathrm{mq}} \simeq 10$ in the absence of a wind $(p=0)$. For a wind with $p=0.4, r_{\mathrm{tr}}=10^{4}$ ("intermediate-wind" model; $\delta=0.3$ ), we find $r_{\mathrm{mq}} \simeq 20$ and $\dot{m}\left(r_{\mathrm{mq}}\right) / \dot{m}\left(r_{\mathrm{tr}}\right) \simeq 8 \times 10^{-2}$, while for a wind with $p=0.8, r_{\mathrm{tr}}=10^{4}$ ("strong-wind" model; $\delta=0.75)$, we find $r_{\mathrm{mq}} \simeq 35$ and $\dot{m}\left(r_{\mathrm{mq}}\right) / \dot{m}\left(r_{\mathrm{tr}}\right) \simeq 1$ $\times 10^{-2}$. For these estimates, we have chosen $r_{\mathrm{tr}}=10^{4}$ by analogy with the values usually inferred in quiescent $\mathrm{BH}$ SXTs (Narayan et al. 1996; Menou et al. 1999). Were $r_{\text {tr }}$ smaller, the overall mass loss in the wind for a given $p$ would be smaller. Note that the ADAF cannot extend far beyond $10^{4}$ Schwarzschild radii because, for a given $\dot{m}\left(r_{\mathrm{tr}}\right)$, there is a maximum radius out to which the ADAF can exist (see, e.g., Esin, McClintock, \& Narayan 1997; Menou et al. 1999).

Following Quataert \& Narayan (1999), we computed two examples of ADAF models assuming that the dominant effect of the wind on the structure and the emission properties of the ADAF is to reduce the density in the accretion 
flow; in addition, we assumed that the emission from the wind itself can be neglected relative to the emission from the accretion flow.

Figure $7 a$ shows the quiescent X-ray luminosities $(0.5-10$ $\mathrm{keV}$ ) of BH SXTs predicted by the ADAF + wind model for which $\dot{m}\left(r_{\mathrm{tr}}\right)$ is equal to $1 / 3$ of the mass supplied by the secondary, $p=0.4, r_{\mathrm{tr}}=10^{4}$, and $\delta=0.3$. This model gives roughly the same $\mathrm{X}$-ray luminosities as the previous no-wind ADAF model (§ 4.1). The actual mass accreting onto the black hole is reduced here, but nevertheless the luminosities are the same because of the increase in the value of $\delta$ from $10^{-3}$ to 0.3 (see Quataert \& Narayan 1999).

Figure $7 b$ shows the quiescent $X$-ray luminosities $(0.5-10$ $\mathrm{keV}$ ) of NS SXTs predicted by the same ADAF + wind model, assuming that all the mass reaching the magnetosphere is accreted onto the NS surface (i.e., no propeller effect). Since the X-ray luminosity of the ADAF is still much less than the luminosity coming from the NS surface, the main effect of including the wind is to decrease the luminosity of quiescent NS SXTs in proportion to the reduction of $\dot{m}$ at the inner edge of the ADAF (eq. [18]). In comparing Figures $4 b$ and $7 b$, it is clear that the luminosity is smaller by roughly an order of magnitude, which is insufficient to explain the observations. Thus, the intermediate-wind scenario must also invoke a propeller effect, though with a somewhat lower efficiency than that described in $\S 5$. Given the uncertainties in our propeller model (see $\S 6.2$ ), we see no objection to such a model.

Only for the strongest wind models $\left(p \sim 1, r_{\mathrm{tr}}=10^{4}\right)$ does the luminosity difference between NS SXTs and BH SXTs predicted by the models reduce to the observed difference. For $p=1$ and $r_{\mathrm{tr}}=10^{4}\left(B_{8}=1, R_{\mathrm{NS}, 6}=1\right)$, equations (18) and (19) predict $r_{\mathrm{mq}} \simeq 58$ and $\dot{m}\left(r_{\mathrm{mq}}\right) / \dot{m}\left(r_{\mathrm{tr}}\right) \simeq 6 \times 10^{-3}$, which is basically consistent with the small accretion rates required to explain the observed quiescent luminosities of NS SXTs (cf. Fig. 4b). However, is this a reasonable model?
Can the propeller effect be completely ineffective at preventing mass from reaching the NS surface?

\subsection{The Efficiency of the Propeller Effect}

If the neutron stars in NS SXTs have spin periods of typically a few milliseconds, as suggested by recent observations (see, e.g., White \& Zhang 1997; Zhang et al. 1998; Campana et al. 1998; Wijnands \& van der Klis 1998), their corotation radii are a few Schwarzschild radii. This is substantially smaller than the few tens of Schwarzschild radii inferred for $r_{\mathrm{mq}}$ in $\S 6.1$. The propeller effect is therefore expected to act quite strongly in these systems (Illarionov \& Sunyaev 1975). Note that the values of $r_{\mathrm{mq}}$ quoted in $\S 6.1$, which correspond to a magnetic field strength $B$ of $10^{8} \mathrm{G}$, would be even larger for a larger $B$ (eq. [19]). Can the propeller in such a system be so ineffective that $f_{\text {acc }} \sim 1$ ?

We pointed out in $\S 5.2$ that there are large uncertainties in the values of $f_{\text {acc }}$, which come mainly from the strong dependence of $f_{\text {acc }}$ on the NS rotation speed and the magnetospheric radius: $f_{\mathrm{acc}} \propto \Omega_{\star}^{-4} R_{\mathrm{mq}}^{-6}$ (the dependence on $R_{\mathrm{mq}}$ also affects the size of the NS emitting area, since $f_{\text {surf }} \propto$ $\left.R_{\mathrm{mq}}^{-4}\right)$. The latter source of uncertainty is perhaps more important, since while $\Omega_{\star}$ can be determined by direct observations, $R_{\mathrm{mq}}$ must be deduced from theoretical arguments.

Although the value of $R_{\mathrm{mq}}$ used in our work (eq. [7]) is standard, it is based on simple dimensional arguments. Given the strong dependence of $f_{\text {acc }}$ on $R_{\mathrm{mq}}$, an error, for instance, of only a factor of 1.5 in $R_{\mathrm{mq}}$ translates to an error of more than an order of magnitude in $f_{\text {acc }}$. Recent work by Psaltis \& Chakrabarty (1998) shows that the standard scaling used for $R_{\mathrm{mq}}$ (for disk-magnetosphere interactions) is not consistent with observations in at least one weakly magnetized NS SXT (SAX J1808.4-3658). Consequently, we cannot rule out the possibility that equation (7) overestimates the value of $R_{\mathrm{mq}}$ for an $\mathrm{ADAF}-$ magnetosphere inter-
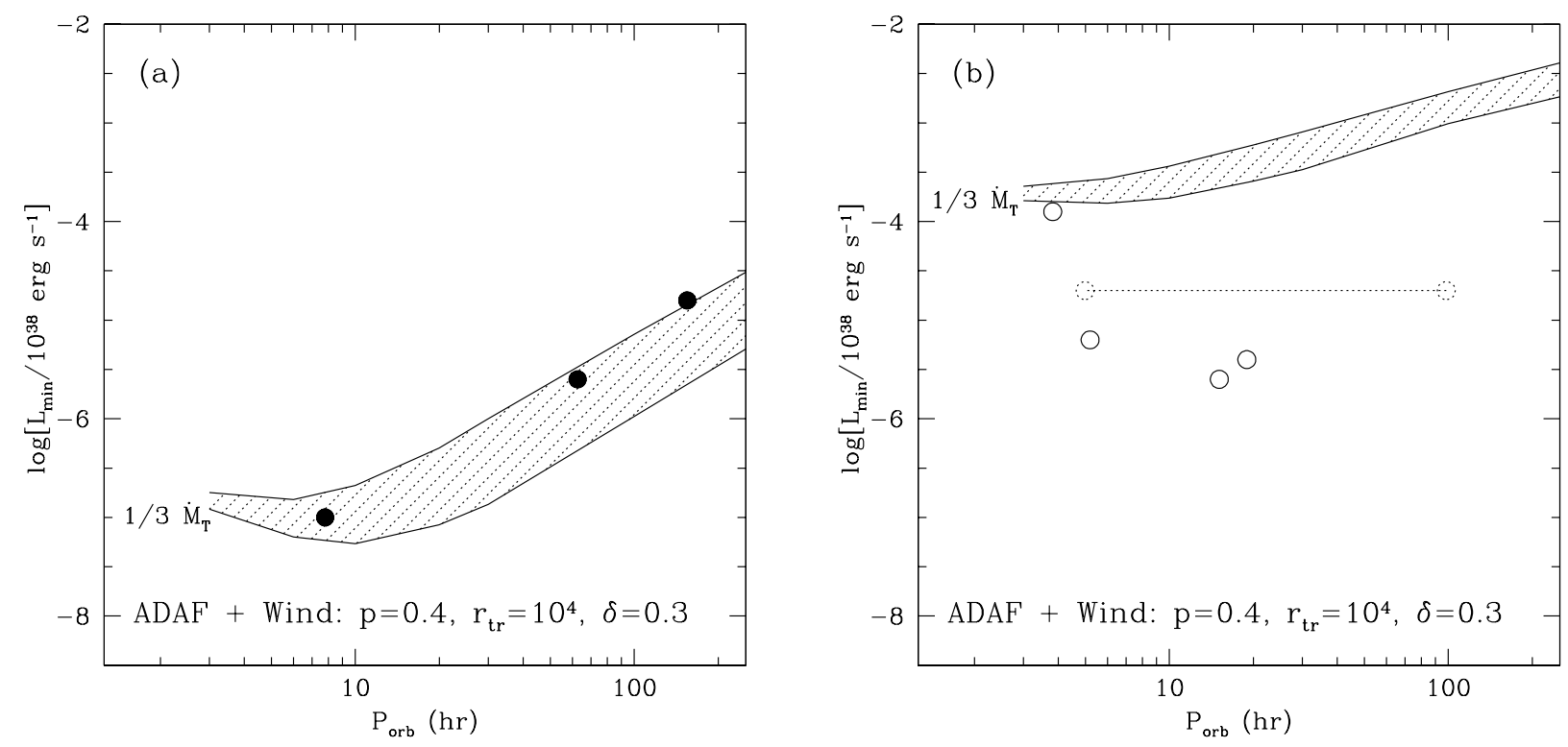

FIG. 7. - (a) band shows the quiescent luminosities of BH SXTs in the $0.5-10 \mathrm{keV}$ band predicted by ADAF models including a wind $\left(p=0.4, r_{\mathrm{tr}}=10^{4}\right.$, and $\delta=0.3$ ) if $1 / 3$ of the mass transferred by the secondary flows into the ADAF. (b) band shows the quiescent luminosities of NS SXTs predicted in the $0.5-10 \mathrm{keV}$ band if (1) $1 / 3$ of the mass transferred by the secondary flows into the ADAF; (2) there is a modest wind $\left(p=0.4, r_{\mathrm{tr}}=10^{4}, \delta=0.3\right)$; and (3) all the mass reaching the NS magnetosphere is accreted onto the surface. The inner radius of the ADAF ( $=$ the magnetospheric radius) is at $r_{\mathrm{mq}}=20$ (see text). The wind reduces, but only by $\sim 1$ order of magnitude, the accretion rate at the magnetosphere (compare with Fig. $4 b$ ). We need a modest propeller to decrease the luminosity further to the observed levels. 
action. The propeller effect would then be significantly less efficient than our previous results suggest $(\S 5)$.

If winds alone $(p \sim 1)$ are to explain the reduced luminosity difference between BH and NS SXTs in quiescence, we need a highly inefficient propeller. For example, from the no-wind $(p=0)$ model to the $p=1$ model described in $\S 6.1$, $r_{\text {mq }}$ increases by a factor $\simeq 5.8$, which implies a $\sim 4 \times 10^{4}$ times more efficient propeller according to equation (11). Since the $p=0$ model has a propeller with $f_{\text {acc }}=10^{-3}$, the propeller would have to be $\sim 10^{7}$ times less efficient in the $p=1$ model than we would infer based on the theory described in $\S 5$. In addition, in the presence of a wind, the value of $f_{\text {acc }}$ is likely only to be smaller than the expression in equation (11). This is because the wind flows out preferentially from the polar regions of the ADAF (Narayan \& Yi 1995a), so there is no gas to flow down to the polar caps of the NS. Even allowing for reasonable uncertainties in the scalings in equation (7), it does not seem likely that the propeller model could be that wrong. We therefore feel that the $p \sim 1$ strong wind model is unlikely.

\section{DISCUSSION}

Chen et al. (1998; their Fig. 3) argued that the segregation in luminosity swing between outburst and quiescence previously found by Narayan et al. (1997b) and Garcia et al. (1998) disappears in a large sample. We point out in this paper that the Chen et al. sample is larger only because they include numerous upper limits, many of which are based on observations with insufficient sensitivity to detect typical quiescent SXTs. Unless treated with care, for instance with a "detection-and-bounds"-type method (see, e.g., Avni et al. 1980; Schmitt 1985), upper limits can obscure a true correlation, and indeed this seems to be the case with the analysis of Chen et al. (1998).

Our Figure 2 shows a carefully selected and smaller sample, and we find that there is a difference of luminosity between quiescent BH and NS SXTs. Furthermore, we argue, based on theoretical mass transfer rates, that it is important to compare quiescent luminosities of NS SXTs and BH SXTs of similar orbital periods. We find that quiescent BH SXTs with short orbital periods are about 2 orders of magnitude fainter (in Eddington units) than quiescent NS SXTs with comparable orbital periods. The difference reduces to a little more than 1 order of magnitude when absolute luminosities are considered. The sample is, however, small. Future observations with the Chandra $X$-Ray Observatory $(C X O)$ and $X M M$ will hopefully increase the sample size sufficiently to provide a more convincing case. Hopefully, they will also allow separate comparisons of long- and short-period systems. This should help solidify the arguments advanced by Narayan et al. (1997b) and Garcia et al. (1998) for event horizons in black holes.

We find that the luminosities of quiescent NS SXTs are lower than the values predicted by standard binaryevolution models when combined with ADAF models. Following Zhang et al. (1998), we argue that the propeller effect offers a plausible explanation for the discrepancy. However, although our model for the propeller effect accounts for the luminosities and small emitting areas of quiescent NS SXTs, it suffers from several sources of uncertainty that may affect the results significantly.

The predictions of the model strongly depend on the spin frequency $\Omega_{\star}$ of the NS and therefore on the prescription chosen for the torque (eq. [15]). The magnitude of the torque is not well known, especially in the propeller regime (see, e.g., Davies et al. 1979; Henrichs 1983). The results of our calculations are also sensitive to the precise values of the magnetospheric radii in quiescence and outburst and to the assumption that the fastness parameter $\omega_{\text {crit }}=1$.

If $\omega_{\text {crit }}<1, \Omega_{\star \text {,eq }}$ is reduced by a factor $\omega_{\text {crit }}$ (eq. [17]) and more mass is accreted because $f_{\text {acc }}$ is $\propto \omega_{\text {crit }}^{-4}$. Note, however, that independently of equation (15) and the value of $\omega_{\text {crit }}$, spin periods of a few milliseconds and low magnetic field strengths (assuming that eq. [7] is correct) lead to predictions for luminosities and surface areas that are consistent with observations.

Even if the spin periods of neutron stars in NS SXTs are known, the predictions of our propeller model are still somewhat uncertain because of the strong dependence of the fractions $f_{\text {acc }}$ and $f_{\text {surf }}$ on the magnetospheric radius in quiescence $R_{\mathrm{mq}}$. If the standard expression (eq. [7]) overestimates the value of $R_{\mathrm{mq}}$, then the propeller effect could be less efficient than indicated by our results in $\S 5$. In that case, winds from ADAFs could provide the additional mechanism required to explain the low quiescent luminosities of NS SXTs.

We have also neglected the likely misalignment between the neutron star spin axis and magnetic axis and have assumed a dipolar structure for the magnetic field. Relaxing these assumptions could also modify our results, especially our estimates of the fractions $f_{\text {acc }}$ and $f_{\text {surf }}$.

The system EXO 0748-676 appears unusually bright in our sample of quiescent NS SXTs. One possible explanation is that magnetic braking acts more efficiently in this system because of its rather short $P_{\text {orb }}$, giving a larger mass transfer rate. If the mass transfer rate is higher, the propeller effect would also be less efficient and would increase the mass falling on the NS even further. Note that EXO $0748-676$ is known to be an unusual binary system, as shown by its unexplained variations of $P_{\text {orb }}$ (Hertz, Wood, \& Cominsky 1997). Another possible explanation is that, for some reason (youth?), this particular system has not yet reached equilibrium and spins at a relatively slower rate than other NS SXTs. This would result in a less efficient propeller and a more luminous system in quiescence.

This last point is a general prediction of our propeller model: the propeller effect is less efficient if the NS in an SXT spins slowly $\left(P_{\text {spin }} \gg\right.$ a few $\left.\mathrm{ms}\right)$. It is possible that several such NS SXTs with slowly spinning NSs exist in our Galaxy. In these NS SXTs, most of the mass accreted via the ADAF would reach the NS in quiescence. The outbursts of these systems would therefore be of small amplitude, 2-3 orders of magnitude in X-rays rather than 5-6 orders of magnitude, because of their relatively high quiescent luminosities.

In our model, the gas which is stopped by the propeller is neglected and its fate is left unspecified. This gas could affect both the dynamics and the emission properties of the accretion flow. For instance, it is unclear how the mass accreted via the ADAF will find its way out of the quasi-spherical accretion flow after being propelled outward.

In $\S 6$, we considered ADAF models with winds assuming that the efficiency $p$ of the wind is the same for BH and NS SXTs . In principle, $p$ could be different for ADAFs around $\mathrm{BHs}$ and those around NSs (because of the presence of the magnetosphere in NSs, for instance). Having a different value of $p$ for BH and NS SXTs would not, however, affect 
our conclusion that winds alone probably cannot explain the observed quiescent luminosities of NS SXTs.

Recently, Brown, Bildsten, \& Rutledge (1998) argued that nuclear reactions in the crusts of neutron stars, triggered during outbursts in NS SXTs, could efficiently heat up the NS cores in these systems. The energy deposited during an outburst would be reemitted during quiescence at a rate sufficient to explain the observed quiescent thermal emission. If so, the propeller effect must be even more efficient than our estimates in $\S 5$ indicate, so that accretion in quiescence does not contribute much to the quiescent emission of NS SXTs.

We note two criteria that bear on the relative importance of crustal heating and accretion to the quiescent luminosity of NS SXTs. First, in the scenario of Brown et al., the entire surface of the NS contributes to the quiescent emission. Small emitting areas have usually been inferred from the observations, by assuming that the quiescent emission is close to blackbody emission. However, Rutledge et al. (1999) have shown that much larger areas are inferred if detailed hydrogen atmosphere models are used instead of idealized blackbody models. There is a large uncertainty in these results because of the poorly known hydrogen column density to the systems. Nevertheless, the values of the emitting areas inferred by Rutledge et al. for several systems are consistent with the entire NS surface being responsible for the emission in quiescence. This success favors the crustal heating model.

On the other hand, any observed rapid variability (e.g., on timescales of a few days) should be considered as strong evidence of the importance of accretion in quiescence, because the intrinsic thermal emission from the NS is not expected to vary on such small timescales (Brown et al. 1998). Campana et al. (1997) reported that the quiescent X-ray luminosity of the prototypical NS SXT Cen X-4 varied by a factor of $\sim 3$ over a period of 4 days. This establishes that the accretion luminosity can be more than 3 times larger than any steady quiescent luminosity possibly present in this system. Though very little X-ray variability data are presently available, several studies of the optical counterpart of Cen X-4 in quiescence have shown a variability of order several tenths of a magnitude (see, e.g., Cowley et al. 1988; Chevalier et al. 1989; McClintock \& Remillard 1990). Since it is natural to relate this optical variability to the X-ray variability observed by Campana et al. (1997), both sets of observations appear consistent with accretion being the dominant mechanism for the quiescent luminosity of NS SXTs.

Throughout this study, we assumed that accretion is responsible for the quiescent emission of both NS and BH SXTs. The possibility that this emission could be due to two different processes in NS SXTs certainly complicates the comparisons between the two classes of systems. We note, however, that even if the dominant contribution to the quiescent emission of NS SXTs is crustal heating, the reason why quiescent NS SXTs, as a class, are brighter than quiescent BH SXTs remains the presence of a hard surface in NS SXTs and of an event horizon in BH SXTs.

\section{CONCLUSION}

In this paper, we have reconsidered the luminosity difference between black hole and neutron star soft X-ray transients in quiescence, which has been used to argue for the presence of event horizons in black holes.
We show that the current observational data suggest that quiescent BH SXTs as a class are fainter than NS SXTs. This result agrees with the previous work of Narayan et al. (1997b) and Garcia et al. (1998), but it disagrees with the conclusions of Chen et al. (1998), who used an inappropriate sample of SXTs for their comparison.

We point out that, for a reliable comparison of the two classes of SXTs, objects with similar orbital periods $P_{\text {orb }}$ should be compared. Otherwise, variations of the mass transfer rate $\dot{M}_{T}$ with $P_{\text {orb }}$ can mask the results.

We find that the observed luminosities of quiescent $\mathrm{BH}$ SXTs are consistent with the predictions of binaryevolution models for $\dot{M}_{T}$ if roughly one-third of the mass supplied by the secondary is accreted by the black hole via an ADAF. This estimate is for $\alpha_{\mathrm{ADAF}}=0.3$. The fraction goes down to $1 / 5$ and $1 / 10$ for $\alpha_{\mathrm{ADAF}}=0.1$ and 0.025 , respectively. The observed luminosities of quiescent NS SXTs suggest, on the other hand, that only a very small fraction of the mass transferred by the secondary reaches the neutron star surface.

We explain the small fraction in NS SXTs by invoking an efficient propeller. We have constructed a model for the propeller effect that accounts for the observed luminosities of quiescent NS SXTs and their small emitting areas. In addition, the model appears to be consistent with the millisecond spin periods recently inferred from observations of accreting neutron stars in transient low-mass binary systems (Aq1 X-1, SAX J1808.4-3658).

Winds from ADAFs constitute an alternative explanation (BB99) for the very small fraction of mass reaching the neutron star surface in quiescence. We argue that an ADAF model with strong winds and no propeller cannot explain the observed luminosities. However, an ADAF model with a wind of low or intermediate strength and a somewhat less efficient propeller (but still within the range of uncertainties of our propeller model) is consistent with the observed quiescent luminosities of BH and NS SXTs. In this case, the parameter $\delta$, which measures the fraction of the viscous energy that goes directly into the electrons, has to be large, $\sim 0.3$ (Quataert \& Narayan 1999).

After submitting our paper for publication, we became aware of an independent paper by Asai et al. (1998) on the same subject. The conclusions of Asai et al. are very similar to those presented here: (1) observational data show evidence for a difference of X-ray luminosity between quiescent BH and NS SXTs, (2) only $0.1 \%-1 \%$ of the mass transferred by the secondary reaches the NS surface in quiescent NS SXTs, and (3) this small accreted fraction could be due to the propeller effect in NS SXTs.

The quiescent fluxes and upper limits reported herein are generally in very good agreement with those listed in Asai et al. (1998), with the exception of $4 \mathrm{U} 1543-47$. Here we assume a distance of $8 \mathrm{kpc}$, while Asai et al. assume $1 \mathrm{kpc}$. This accounts for most, but not all of the discrepancy between the Asai et al. upper limit and that in Orosz et al. (1998). We choose to use the latter limit, as it is more conservative.

We are grateful to Josh Grindlay, Mario Livio, Philip Podsiadlowski, Dimitrios Psaltis, and Eliot Quataert for useful discussions, and to an anonymous referee for constructive comments. A. E. and R. N. also gratefully acknowledge their conversation with Shuang-Nan Zhang, who suggested the idea of a propeller effect in the context of a 
quasi-spherical accretion flow. This work was supported in part by NASA grant NAG 5-2837. K. M. was supported by a Smithsonian Astrophysical Observatory (SAO) Predoctoral Fellowship and a French Higher Education Ministry Grant. A. E. was supported by a National Science Founda- tion Graduate Research Fellowship and by NASA through $A X A F$ Fellowship grant PF8-10002 awarded by the $A X A F$ Science Center, which is operated by the SAO for NASA under contract NAS8-39073.

\section{REFERENCES}

Abramowicz, M., Chen, X.-M., Granath, M., \& Lasota, J.-P. 1996, ApJ, 471,762

Abramowicz, M., Chen, X.-M., Kato, S., Lasota, J.-P., \& Regev, O. 1995, ApJ, 438, L37

Asai, K., et al. 1998, PASJ, 50, 611

Avni, Y., Soltan, A., Tananbaum, H., \& Zamorani, G. 1980, ApJ, 238, 800

Bailyn, C. D., Jain, R. K., Coppi, P., \& Orosz, J. A. 1998, ApJ, 499, 367

Beekman, G., et al. 1997, MNRÄS, 290, 303

Bisnovatyi-Kogan, G. S., \& Lovelace, R. V. E. 1997, ApJ, 486, L43

Blandford, R. D., \& Begelman, M. C. 1999, MNRAS, 303, L1 (BB99)

Bondi, H. 1952, MNRAS, 112, 195

Brown, E. F., Bildsten, L., \& Rutledge, R. E. 1998, ApJ, 504, L95

Campana, S., Mereghetti, S., Stella, L., \& Colpi, M. 1997, A\&A, 324, 941

Campana, S., et al. 1998, ApJ, 499, L65

Cannizzo, J. K. 1993, in Accretion Disks in Compact Stellar Systems, ed.

J. C. Wheeler (Singapore: World) 6

Chakrabarty, D., \& Morgan, E. H. 1998, Nature, 394, 346 (CM98)

Chen, W., Shrader, C. R., \& Livio, M. 1997a, ApJ, 491, 312

Chen, W., et al. 1998, in AIP Conf. Proc. 431, Accretion Processes in Astrophysics: Some Like it Hot, ed. S. Holt \& T. Kallman (Woodbury, NY: AIP), 347

Chen, X. M., Abramowicz, M. A., \& Lasota, J.-P. 1997b, ApJ, 476, 61

Chevalier, C., Ilovaisky, S. A., van Paradijs, J., Pedersen, H., \& van der

Klis, M. 1989, A\&A, 210, 114

Cowley, A. P., et al. 1988, AJ, 95, 1231

Cui, W. 1997, ApJ, 482, L163

Cui, W., Barret, D., Zhang, S. N., Chen, W., Boirin, L., \& Swank, J. 1998, ApJ, 502, L49

Daumerie, P. R. 1996, Ph.D. thesis, Univ. Illinois, Urbana-Champaign

Davies, R. E., Fabian, A. C., \& Pringle, J. E. 1979, MNRAS, 186, 779

Davies, R. E., \& Pringle, J. E. 1981, MNRAS, 196, 209

Esin, A. A., McClintock, J. E., \& Narayan, R. 1997, ApJ, 489, 865

Esin, A. A., Narayan, R., Cui, W., Grove, J. E., \& Zhang, S.-N. 1998, ApJ, 505,854

Frank, J., King, A., \& Raine, D. 1992, Accretion Power in Astrophysics (Cambridge: Cambridge Univ. Press)

Garcia, M. R., McClintock, J. E., Narayan, R., \& Callanan, J. 1998, in ASP Conf. Ser. 137, Wild Stars in the Old West, Proc. 13th North American Workshop on Cataclysmic Variables, ed. S. Howell, E. Kuulkers, \& C. Woodward (San Francisco: ASP), 506

Ghosh, R., \& Lamb, F. K. 1979, ApJ, 234, 296

Gruzinov, A. 1998, ApJ, 501, 787

Hameury, J.-M., Lasota, J.-P., McClintock, J. E., \& Narayan, R. 1997, ApJ, 489,234

Henrichs, H. F. 1983, in Accretion-driven Stellar X-Ray Sources, ed. W. H. G. Lewin \& E. P. J. van den Heuvel (Cambridge: Cambridge Univ. Press), 393

Hertz, P., Wood, K. S., \& Cominsky, L. R. 1997, ApJ, 486, 1000

Ichimaru, S. 1977, ApJ, 214, 840

Illarionov, A. F., \& Sunyaev, R. A. 1975, A\&A, 39, 185

Kalogera, V., Kolb, U., \& King, A. 1998, ApJ, 504, 967

Kato, S., Fukue, U., \& Mineshige, S. 1998, Black Hole Accretion Disks (Kyoto: Kyoto Univ. Press)

King, A. 1988, QJRAS, 29, 1

King, A., Kolb, U., \& Burderi, L. 1996, ApJ, 464, L127

Kolb, U. 1998, MNRAS, 297, 419

Kuulkers, E. 1998, NewAR, 42, 1

Lasota, J.-P. 1996, in IAU Symp. 165, Compact Stars in Binaries, ed. J. van Paradijs, E. P. J. van den Heuvel, \& E. Kuulkers (Dordrecht: Kluwer), 43

Lasota, J.-P., \& Hameury, J.-M. 1998, in AIP Conf. Proc. 431, Accretion Processes in Astrophysics: Some Like it Hot, ed. S. Holt \& T. Kallman (Woodbury, NY: AIP), 351

Lasota, J.-P., Narayan, R., \& Yi, I. 1996, A\&A, 314, 813

Lewin, W. H. G., van Paradijs, J., \& Taam, R. E. 1993, Space Sci. Rev., 62, 223

McClintock, J. E. 1998, in AIP Conf. Proc. 431, Accretion Processes in Astrophysics: Some Like it Hot, ed. S. Holt \& T. Kallman (Woodbury, NY: AIP), 290
McClintock, J. E., \& Remillard, R. A. 1990, ApJ, 350, 386

Menou, K., Narayan, R., \& Lasota, J.-P. 1999, ApJ, 513, 811

Meyer, F., \& Meyer-Hofmeister, E. 1994, A\&A, 288, 175

Nakamura, K. E., Kusunose, M., Matsumoto, R., \& Kato, S. 1997, PASJ, 49,503

Narayan, R., Barret, D., \& McClintock, J. E. 1997a, ApJ, 482, 448

Narayan, R., Garcia, M. R., \& McClintock, J. E. 1997b, ApJ, 478, L79

Narayan, R., Kato, S., \& Honma, M. 1997c, ApJ, 476, 49

Narayan, R., Mahadevan, R., Grindlay, J. E., Popham, R. G., \& Gammie, C. F. 1998, ApJ, 492, 554

Narayan, R., Mahadevan, R., \& Quataert, E. 1999, in The Theory of Black Hole Accretion Discs, ed. M. A. Abramowicz, G. Bjornsson, \& J. E. Pringle (Cambridge: Cambridge Univ. Press), in press (astro-ph 9803141)

Narayan, R., McClintock, J. E., \& Yi, I. 1996, ApJ, 457, 821

Narayan, R., \& Yi, I. 1994, ApJ, 428, L13

. 1995a, ApJ, 444, 231

.1995b, ApJ, 452, 710

Orosz, J. A., \& Bailyn, C. D. 1997, ApJ, 477, 876

Orosz, J. A., Jain, R. K., Bailyn, C. D., McClintock, J. E., \& Remillard, R. A. 1998, ApJ, 499, 375

Phillips, S. N., Shahbaz, T., \& Podsiadlowski, P. 1999, MNRAS, in press (astro-ph 9811474)

Popham, R. G., \& Gammie, C. F. 1998, ApJ, 504, 419

Psaltis, D., \& Chakrabarty, D. 1999, ApJ, in press

Pylyser, E., \& Savonije, G. J. 1988, A\&A, 191, 57

Quataert, E. 1998, ApJ, 500, 978

Quataert, E., \& Gruzinov, A. 1999, ApJ, in press

Quataert, E., \& Narayan, R. 1999, ApJ, in press

Rajagopal, M., \& Romani, R. W. 1996, ApJ, 461, 327

Ritter, H., \& Kolb, U. 1998, A\&AS, 129, 83

Rutledge, R. E., Bildsten, L., Brown, E. F., Pavlov, G. G., \& Zavlin, V. E. 1999, ApJ, 514, 945

Schmitt, J. H. M. 1985, ApJ, 293, 178

Shakura, N. I., \& Sunyaev, R. A. 1973, A\&A, 24, 337

Shapiro, S. L., \& Salpeter, E. E. 1975, ApJ, 198, 671

Shaviv, G., \& Wehrse, R. 1986, A\&A, 159, L5

Stella, L., Campana, S., Colpi, M., Mereghetti, S., \& Tavani, M. 1994, ApJ, 423, L47

Stella, L., White, N. E., \& Rosner, R. 1986, ApJ, 308, 669

Tanaka, Y., \& Lewin W. H. G. 1995, in X-Ray Binaries, ed. W. H. G. Lewin, J. van Paradijs, \& E. P. J. van den Heuvel (Cambridge: Cambridge Univ. Press), 126

Tanaka, Y., \& Shibazaki, N. 1996, ARA\&A, 34, 607

Turolla, R., Zampieri, L., Colpi, M., \& Treves, A. 1994, ApJ, 426, L35

van Paradijs, J. 1995, in X-Ray Binaries, ed. W. H. G. Lewin, J. van Paradijs, \& E. P. J. van den Heuvel (Cambridge: Cambridge Univ. Press), 536

van Paradijs, J., \& McClintock, J. E. 1995, in X-Ray Binaries, ed. W. H. G. Lewin, J. van Paradijs, \& E. P. J. van den Heuvel (Cambridge: Cambridge Univ. Press), 58

Verbunt, F., Belloni, T., Johnston, H. M., van der Klis, M., \& Lewin, W. H. G. 1994, A\&A, 285, 903

Verbunt, F., \& van den Heuvel, E. P. J. 1995, in X-Ray Binaries, ed. W. H. G. Lewin, J. van Paradijs, \& E. P. J. van den Heuvel (Cambridge: Cambridge Univ. Press), 457

Wang, Y.-M. 1987, A\&A, 183, 257

. 1995, ApJ, 449, L153

Wang, Y.-M., \& Robertson, J. A. 1985, A\&A, 151, 361

Warner, B. 1995, Cataclysmic Variable Stars (Cambridge: Cambridge Univ. Press)

Webbink, R. F., Rappaport, S., \& Savonije, G. J. 1983, ApJ, 270, 678

White, N. E., Nagase, F., \& Parmar, A. N. 1995, in X-Ray Binaries, ed. W. H. G. Lewin, J. van Paradijs, \& E. P. J. van den Heuvel (Cambridge: Cambridge Univ. Press), 1

White, N. E., \& Zhang, W. 1997, ApJ, 490, L87

Wijnands, R., \& van der Klis, M. 1998, Nature, 394, 344

Zavlin, V. E., Pavlov, G. G., \& Shibanov, Y. A. 1996, A\&A, 331, 821

Zhang, S. N., Yu, W., \& Zhang, W. 1998, ApJ, 494, L71 